\title{
MAP: Multiauctioneer Progressive Auction for Dynamic Spectrum Access
}

\author{
Lin Gao, Student Member, IEEE, Youyun Xu, Member, IEEE, and Xinbing Wang, Member, IEEE
}

\begin{abstract}
Cognitive radio $(C R)$ is a promising paradigm to achieve efficient utilization of the limited spectrum resource by allowing the unlicensed users to access the licensed spectrum, and dynamic spectrum access (DSA) is one of the fundamental functions of CR networks. Market-driven spectrum auction has been recognized as an effective way to achieve DSA. In spectrum auction, the primary spectrum owners (POs) act as auctioneers who are willing to sell idle spectrum bands for additional revenue, and the secondary users (SUs) act as bidders who are willing to buy spectrum bands from POs for their services. However, conventional spectrum auction designs are restricted within the scenario of single auctioneer. In this paper, we study the spectrum auction with multiple auctioneers and multiple bidders, which is more realistic for practical CR networks. We propose MAP, a Multiauctioneer Progressive auction mechanism, in which each auctioneer systematically raises the trading price and each bidder subsequently chooses one auctioneer for bidding. The equilibrium is defined as the state that no auctioneer and bidder would like to change his decision. We show analytically that MAP converges to the equilibrium with maximum spectrum utilization of the whole system. We further analyze the incentive for POs and SUs joining the auction and accepting the auction result. Simulation results show that MAP well converges to the equilibrium, and the spectrum utilization is arbitrary closed to the global optimal solution according to the length of step.
\end{abstract}

Index Terms-Cognitive radio, dynamic spectrum access, auction theory, spectrum auction, dual theory.

\section{INTRODUCTION}

$\mathrm{C}$ URRENTLY, wireless communication systems suffer from scarcity in spectrum resource and inefficiency in spectrum usage. Cognitive radio (CR) or dynamic spectrum access (DSA) [3], [4], [5] has been viewed as a novel approach for improving the spectrum utilization by allowing the unlicensed users (i.e., secondary users, SUs) to access the licensed spectrum in an opportunistic manner. Through DSA, the SUs have a chance to employ the idle spectrum which impliedly improving the spectrum efficiency, and the primary spectrum owners (POs) can gain potential profits by leasing their idle spectrum temporarily. To realize this, it is essential to design a spectrum assignment mechanism by which all idle spectrum can be efficiently assigned to the SUs.

In this paper, we consider the problem of spectrum assignment among SUs in CR networks with multiple POs. Each PO owns a set of residual spectrum bands (idle channels) which can be shared by SUs, and each SU has a desire to employ spectrum band for his services. We address the following essential issues: 1) how to assign the residual spectrum bands among the SUs with the highest spectrum utilization, and 2) what is the motivation for each $P O$ and SU accepting such an assignment. The first issue is referred to as efficiency and the second as incentive.

- L. Gao is with the Institute of Wireless Communication Technology, Shanghai Jiao Tong University, No. 800 Dongchuan Road, Shanghai, China 200240, and also with the State Key Laboratory of Integrated Services Networks, Xidian University. E-mail: gaull@sjtu.edu.cn.

- Y. Xu and X. Wang are with the Institute of Wireless Communication Technology, Shanghai Jiao Tong University, No. 800 Dongchuan Road, Shanghai, China 200240. E-mail: \{xuyouyun, xwang8\}@sjtu.edu.cn.

Manuscript received 13 July 2009; revised 24 Oct. 2009; accepted 12 Aug. 2010; published online 15 Nov. 2010.

For information on obtaining reprints of this article, please send e-mail to: tmc@computer.org, and reference IEEECS Log Number TMC-2009-07-0288. Digital Object Identifier no. 10.1109/TMC.2010.220.
In general, an efficient spectrum assignment can be achieved by centralized method such as linear programming based branch-and-bound algorithm [21] or graph theory-based optimal matching algorithm [22]. However, the centralized approaches are not suitable for CR networks due to the distributed nature of $C R$ networks and the heterogeneous nature of both POs and SUs (see Section 3.3 for detail). Market-driven auction has been recognized as an effective mechanism to allocate resources in a distributed manner [11], thus we introduce the concept of spectrum auction into this work.

In spectrum auction, the POs act as auctioneers who own the idle spectrum bands and sell them to SUs for additional revenue, and the SUs act as bidders who buy spectrum bands from POs for their services. In essence, spectrum auction is a combination of the spectrum assignment process and profit transfer process. Unfortunately, conventional spectrum auction designs fall into the scope of oneshot auction which is usually restricted within the scenario of single auctioneer. Since the practical CR networks usually contain multiple POs, it is more realistic to study the spectrum auction with multiple auctioneers. For this purpose, we propose a multishot auction named as Multiauctioneer Progressive auction (MAP). The main difference between MAP and conventional spectrum auctions is that MAP allows for multiple auctioneers each making the decision independently. To our best knowledge, there is no work yet which applies auction with multiple auctioneers to resource allocation problems in wireless networks.

The main contributions of the article are as follows:

- We design the detail mechanism of MAP, which works in a totally distributed manner and can be effectively implemented in the system with multiple POs. The main idea of MAP is that: each auctioneer systematically raises the trading price and each 
bidder subsequently decides whether to buy the spectrum band and from which PO he is going to buy.

- We define equilibrium as the state that no auctioneer and bidder would like to deviate from his decision. We show that the equilibrium exists, and MAP converges to the equilibrium.

- We prove that MAP achieves the optimal spectrum assignment using dual theory. We further show that, through the inherent profit transfer process in auction mechanism, both POs and SUs are willing to accept the assignment achieved by MAP.

- We discuss in detail the distributed implementation of MAP protocol in practical networks, from the aspects of time synchronizing, sensing and decoding, signal interacting, step determining, etc.

The paper is organized as follows: In Section 2, we present the related work on spectrum assignment in CR networks. In Section 3, we introduce the system model of CR networks with multiple POs and SUs. In Section 4, we address the optimal spectrum assignment problem in a centralized manner. In Section 5, we provide an auction-based spectrum assignment mechanism, MAP. In Section 6, we discuss convergence properties of MAP and provide our simulation results. Finally, we conclude our work in Section 7.

\section{Related Work}

As an intelligence extension of software-defined radio (SDR) [2], cognitive radio [3] has been proposed as the promising means to promote the efficient use of the spectrum. There are two comprehensive surveys [4], [5] on cognitive radio networks and dynamic spectrum access, respectively. In [4], Akyildiz et al. identified four fundamental tasks of cognitive radio networks: spectrum sensing, spectrum management, spectrum sharing and spectrum mobility, and dynamic spectrum access in fact involves all of them. In [5], Zhao et al. categorized the existing work on dynamic spectrum access into three general models: dynamic exclusive use model, open sharing model, and hierarchical access model.

Many approaches for dynamic spectrum access in cognitive radio networks were proposed, including graphtheoretic approaches [6], [7], game-theoretic approaches [8], [9] and [27] so forth. In [10], an optimization problem was formulated for dynamic spectrum access to achieve highest utility for SUs with the constraints of quality of service (QoS) and interference temperature, which could be applied to the wireless sensor networks [18].

Auction is an efficient mechanism to allocate resources in market [11], and has been viewed as a promising approach to allocate spectrum in wireless networks [12]. Some of the recent work on spectrum auction designs are as follows: In [13], Huang et al. proposed two pricing schemes for auction-based power allocation to achieve efficiency (social optimality) and fairness, respectively. In [14], Gandhi et al. formulated a general spectrum auction problem as an optimization problem and emphasized on the choice of market clearing price. Truthfulness (or strategy-proofness) was addressed in [15], [16], [17]. In [15], Li et al. proposed several algorithms to assign spectrum such that the social efficiency are approximately maximized, and then designed strategy-proof mechanisms to charge the SUs. In [16], Zhou et al. proposed a truthful single-side auction called VERITAS for spectrum auctions characterized by heterogeneity and spectrum reuse among wireless devices. In [17], Zhou and Zheng, proposed a general framework for truthful double spectrum auctions called TRUST, wherein an external auctioneer with complete information runs the auction to enable the POs and SUs to trade spectrum dynamically. However, the above spectrum auctions are all restricted within the scenario of single auctioneer, either acted by a PO in single PO networks or a virtual centralized entity in multi PO networks. We study the spectrum auction in multi PO CR networks without any centralized entity, wherein each $\mathrm{PO}$ acts as an individual auctioneer and makes his decision independently.

\section{System Model and Problem Formulation 3.1 System Description}

We consider a CR network consisting of $M$ POs and $N$ SUs, and we allow the POs (or SUs) to be heterogeneous. We assume there is no centralized control entity in the network, and thus there is no collaboration among POs (or SUs) since it is very difficult to collaborate the heterogeneous POs (or SUs) without any centralized entity. Each PO serves a set of subscribed primary users and the spectrum bands it possessing may be under utilized, i.e., there may exist some residual spectrum bands (also called residual channels or idle channels) which are not used by primary users at any particular time. Therefore, the PO allows the SUs' to opportunistically access those residual channels to gain potential profit.

We denote the number of residual channels owned by PO $i$ as $m_{i}$, and we assume $m_{i}$ is constant in the time period of interest. ${ }^{1}$ In fact, $m_{i}$ is mainly determined by the number of channels $\mathrm{PO} i$ owns and the pattern of primary users' channel occupancy in PO $i$. While in this work we focus on a static wireless environment, we can easily extend to the dynamic wireless environment with non constant $m_{i}$. Specifically, in the latter case, we can divide the whole time period into small enough time intervals such that $m_{i}$ is constant in each interval.

We assume that channels owned by the same PO are identical, that is, they have the same bandwidth, carrier frequency, coding and modulating scheme, etc., whereas the channels in different POs may be different due to the heterogeneities of POs. We denote the carrier frequency and bandwidth of channels in PO $i$ as $f_{i}$ and $w_{i}$, respectively. Considering a GSM-based PO $i$, we have $f_{i}=900 \mathrm{MHz}$ (or $1800 \mathrm{MHz}$ ) and $w_{i}=200 \mathrm{KHz}$.

We assume there is no spectrum reuse among the SUs, which implies each SU can hear (and interfere) the transmissions of other SUs if they are using the same channel. ${ }^{2}$ Thus we have the first constraint: each channel can only be used by one SU at the same time. In addition, advanced spectrum aggregation techniques are still immature and it

1. Note that throughout the paper, we use superscript $i$ to denote a variable associated with PO $i$ and subscript $n$ for SU $n$.

2. This assumption is obviously available for the network within any small region, e.g., a building. For wide and open region, the assumption is also available if the SUs work as virtual primary users and access the network through the primary base stations. 


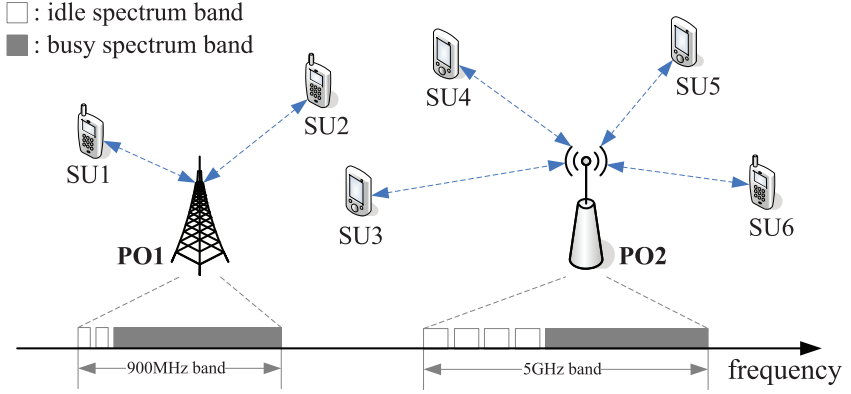

Fig. 1. An example of CR network with 2 POs and 6 SUs.

is difficult for a radio device to employ multiple discontiguous spectrum bands, whether these spectrum bands are possessed by the same PO or different POs. Thus we have the second constraint: each SU can only use one channel at a particular time.

Fig. 1 presents an example of CR network with 2 POs and 6 SUs, where PO1 and PO2 operate on the frequency of $900 \mathrm{MHz}$ (e.g., GSM-based system) and $5 \mathrm{GHz}$ (e.g., IEEE 802.11a-based system), respectively. The gray bars in frequency-axes denote the busy spectrum bands which are being used by the primary users, and the white bars denote the residual spectrum bands (idle channels) owned by each $\mathrm{PO}$ at the moment. Obviously, there are two residual channels owned by PO1 and 4 residual channels owned by $\mathrm{PO} 2$. If we assume that PO1 operates in a GSM-based system and PO2 operates in a 802.11a-based system, the bandwidth of channel owned by PO1 and PO2 are $200 \mathrm{KHz}$ and $20 \mathrm{MHz}$, respectively. Each dashed line between POs and SUs indicates that the SU employs a residual channel of the associated PO. We can easily find that SU1 and SU2 employ channels of PO1 and other SUs employ channels of PO2.

To facilitate the reading, we list the major notations used in our model and analysis in Table 1.

\subsection{Reservation Price and Valuation}

If a $\mathrm{PO}$ accepts an accessing request of any $\mathrm{SU}$, he will assign an idle channel to the SU and serve the SU on that channel. We call this process "PO assigning a channel to $\mathrm{SU}^{\prime \prime}$ for short. ${ }^{3}$ We denote the assignment from PO $i$ to SU $n$ as $(n, i)$. In each assignment, the PO suffers certain expense and the $\mathrm{SU}$ gains certain benefit.

For each $\mathrm{PO}$, we define the reservation price (also called cost) of a residual channel as the expense of serving the assigned SU on that channel. This reservation price represents the disadvantageous impact of SUs' transmission on POs' performance, which may consist of power consumption, device depreciation, performance degradation of primary users, etc. We denote the reservation price of $\mathrm{PO} i$ as $c_{i}$. The reservation price vector, denoted by $\mathbf{c}$, is defined by all POs' reservation prices:

$$
\mathbf{c}=\left(c_{1}, c_{2}, \ldots, c_{M}\right) .
$$

For each SU, we define the valuation for a channel as the benefit of using that channel. This valuation is often

3. For simplicity, we will use the following statements interchangeably in this paper: 1) PO $i$ assigns a channel to SU $n, 2)$ PO $i$ serves SU $n$ on a channel, 3) SU $n$ employs (utilizes) a channel of PO $i$, and 4) there is an assignment from $\mathrm{PO} i$ to $\mathrm{SU} n$.
TABLE 1

Notations

\begin{tabular}{|ll|}
\hline$\epsilon$ & The trading price update step size of POs \\
$f_{i}$ & The carrier frequency of PO $i$ \\
$w_{i}$ & The bandwidth of channels of PO $i$ \\
$m_{i}$ & The number of residual channels of PO $i$ \\
$c_{i}$ & The reservation price of $\mathrm{PO} i$ \\
$p_{i}$ & The trading price of PO $i$ \\
$d_{i}$ & The demand for channels owned by PO $i$ \\
$\mathbf{y}_{i}$ & The strategy of PO $i$ \\
$v_{n i}$ & The valuation of $\mathrm{SU} n$ for the channel of $\mathrm{PO} i$ \\
$x_{n i}$ & The interest of SU $n$ for buying the channel from $\mathrm{PO} i$ \\
$\mathbf{x}_{n}$ & The strategy of SU $n$ \\
$r_{n i}$ & The assignment between $\mathrm{PO} i$ and SU $n$ \\
\hline
\end{tabular}

related to the channel capacity or channel quality (typically signal-to-noise ratio, SNR). Since the channels within the same PO are identical, we simply denote the valuation of SU $n$ for the channel of PO $i$ as $v_{n i}$. We can generally model $v_{n i}$ as a function of the channel bandwidth and SNR: $v_{n i}=\mathcal{G}_{n}\left(\Gamma_{n i}, w_{i}\right)$, where $\Gamma_{n i}$ is the channel SNR between SU $n$ and PO $i$, and $\mathcal{G}_{n}(x, y)$ is a nonnegative and nondecreasing function with respect to $x$ and $y$. Similarly, the valuation matrix, denoted by $\mathbb{W}$, is composed of all SUs' valuations for all channels:

$$
\mathbb{W}=\left(\begin{array}{c}
\mathbf{v}_{1} \\
\cdots \\
\mathbf{v}_{N}
\end{array}\right),
$$

where $\mathbf{v}_{n}=\left(v_{n 1}, v_{n 2}, \ldots, v_{n M}\right)$ is the valuation vector of $\mathrm{SU} n$ for all POs' channels.

It is worth noting that $c_{i}$ is private information of $\mathrm{PO} i$ and $v_{n i}$ is private information of $\mathrm{SU} n$. For any $\mathrm{PO}$ or $\mathrm{SU}$, it is very hard to obtain the private information of others in a noncooperative network. As will be discussed later, this is also a reason that we try to solve the problem by means of auction theory, since auction is essentially a game with incomplete information [11].

It is also notable that the valuation of SU for any channel is not only related to the channel bandwidth and SNR, but also to the service type. In fact, each SU $n$ can select different form of $\mathcal{G}_{n}(x, y)$ for different services. For example, for voice services which sensitive to delay and bit-errorratio (BER), the SU can model the valuation as a sigmoid function with respect to SNR. For data services which sensitive to bandwidth and bit rate, the SU can model the valuation as the Shannon Capacity [1]. Further, if any PO's transmission mode is not supported by SU, e.g., an OFDMbased PO and a CDMA-based SU, the SU can simply set the associated valuation to zero since he cannot use those channels at all.

\subsection{Spectrum Assignment Problem Formulation}

According to definition of valuation, we can easily find that different SUs may have different valuations for the same channel and different channels may have different values for the same SU. Besides, according to the previous assumptions, each SU can only use one channel and each channel can only be used by one SU. Thus we address the following spectrum assignment problem: 1) how to assign the 
$\sum_{i \in M} m_{i}$ residual channels among all SUs to achieve the highest spectrum utilization, ${ }^{4}$ and 2) what is the motivation for each $P O$ and SU to accept such an assignment. The first issue is referred to as efficiency and the second as incentive.

We define the channel assignment matrix as an $N \times M$ matrix $\mathbb{R}=\left\{r_{n i}\right\}_{N \times M}$ where each element $r_{n i} \in\{0,1\}$ indicates whether PO $i$ assigns one residual channel to $\mathrm{SU} n$, that is, $r_{n i}=1$ denotes that PO $i$ assigns a channel to SU $n$ and $r_{n i}=0$ otherwise. It is easy to see that a feasible channel assignment matrix must satisfy the following constraints: 1) $\sum_{i \in M} r_{n i} \leq 1$, since each SU can only use one channel, and 2) $\sum_{n \in N} r_{n i} \leq m_{i}$, since each channel can only be used by one SU.

If PO $i$ assigns a channel to SU $n$, i.e., $r_{n i}=1$, the PO suffers expense $c_{i}$ and the SU gains profit $v_{n i}$, thus we define net profit (or social profit), denoted by $s_{n i}$, as the difference of $\mathrm{SU}^{\prime} \mathrm{s}$ valuation and $\mathrm{PO}^{\prime}$ s expense, i.e., $s_{n i}=v_{n i}-c_{i}$. In fact, $s_{n i}$ represents the increase of social wealth brought by the assignment from PO $i$ to SU $n$. Further, we define the social income as the aggregate net profits of all assignments in $\mathbb{R}$. The social income is also regarded as the spectrum utilization of whole network. Formally, we can write the social income as follows:

$$
S=\sum_{n \in N} \sum_{i \in M} r_{n i} \cdot\left(v_{n i}-c_{i}\right) .
$$

An optimal channel assignment refers to a feasible channel assignment which maximizes the social income $S$. Formally, we can write the optimal channel assignment as the following optimization function:

$$
\begin{aligned}
\mathbb{R}^{*}=\arg \max _{\mathbb{R}} S=\arg \max _{\mathbb{R}} \sum_{n \in N} \sum_{i \in M} r_{n i} \cdot\left(v_{n i}-c_{i}\right) \\
\text { s.t. (i) } \sum_{i \in M} r_{n i} \leq 1, \forall n \in N, \\
\text { (ii) } \sum_{n \in N} r_{n i} \leq m_{i}, \forall i \in M, \\
\text { (iii) } r_{n i} \in\{0,1\}, \forall i \in M, n \in N .
\end{aligned}
$$

In fact, (4) defines a binary linear programming (BIP) problem, a special case of integer programming in which variables can only be 0 or 1 . The BIP problem is NP-hard in general. We can use Branch-and-Bound algorithm [21] to solve this problem, or we can transform the original problem into the optimal matching problem in graph theory [20] and use Kuhn-Munkres algorithm [22] to solve this problem. However, both of the algorithms fall into the scope of centralized algorithm which is not suitable for the distributed and noncooperative $\mathrm{CR}$ networks due to the following two reasons.

On one hand, the centralized algorithm needs complete information, while in our model the reservation price and valuation are private information for POs and SUs, respectively. As mentioned previously, it is hard to obtain the private information of others in noncooperative and distributed CR networks. On the other hand, even if there is a centralized entity who successfully collects the complete information, the selfish SUs may not accept the optimal channel assignment. In other words, the centralized

4. We write the expression $i \in\{1,2, \ldots, M\}$ as $i \in M$ for simplicity. algorithm cannot provide the incentive for SUs. We show this problem by an example in the network with 2 POs and 2 SUs where $c_{1}=c_{2}=0, m_{1}=m_{2}=1, \mathbf{v}_{1}=(5,1)$ and $\mathbf{v}_{2}=$ $(4,2)$. Obviously the optimal channel assignment is $r_{11}^{*}=$ $r_{22}^{*}=1$ and $r_{12}^{*}=r_{21}^{*}=0$, that is, SU1 employs the channel of PO1 and SU2 employs the channel of PO2. However, the $\mathrm{SU} 2$ will disobey the assignment since $v_{21}>v_{22}$ !

\subsection{Spectrum Auction Description}

Auction has been recognized as an effective mechanism to allocate resources in wireless networks in a distributed manner [11]. Additionally, the CR networks with multiple POs and SUs can be easily regarded as a two-sided market. Thus it is natural to apply auction mechanism for channel assignment problem in CR networks.

In spectrum auction, the POs act as auctioneers who sell their residual channels to SUs, and the SUs act as bidders who buy channels from POs. In other words, if an SU employs a channel of any PO, he must pay the PO a given monetary payment for using that channel. Such a payment is called the trading price of the channel. Note that each assignment is associated with a trading price which can be specified either by the associated PO or SU. From the economic perspective, $c_{i}$ is the minimum trading price at which PO $i$ is willing to sell a residual channel, and $v_{n i}$ is the maximum trading price at which $\mathrm{SU} n$ is willing to pay for a channel of PO $i$.

The SUs gain the profit by using the POs' channels for their services and the POs also gain the profit by charging SUs the trading price. In essence, spectrum auction is a combination of conventional channel assignment process and profit transfer process. For simplicity, we list the key elements of spectrum auction as follows:

1. Bidders: all SUs desiring to employ channels;

2. Auctioneers: all POs willing to sell residual channels;

3. Auctioned Items: all residual channels.

It is easy to see that our spectrum auction falls into the scope of multiauctioneer multiitem auction.

According to the difference of bidding behavior, auctions can be divided into two categories: one-shot auction and multishot auction [11]. In one-shot auction, all the bidders submit their bids to auctioneer simultaneously and the auctioneer sets the trading price at once, taking into account the bids collectively. In multishot auction, the auctioneer dynamically adjusts the trading price and at the instance when price changes the bidders react by, for example showing whether or how many they are willing to buy from the auctioneer. Thus one-shot auction is also referred to as static auction and multishot auction as dynamic or progressive auction.

From the aspect of bidding behavior, one-shot auction works in a centralized or quasi-centralized manner. For market with single seller (i.e., single PO), one-shot auction can be directly implemented since the seller can work as the central processing entity. Specifically, the bidders submit their bids to the seller and the seller works out the marketclearing price in a centralized manner (e.g., see examples of one-shot auction in [15], [16]). While for market with multiple sellers (i.e., multiple POs), one-shot auction cannot be directly implemented. In one hand, the bidders cannot 


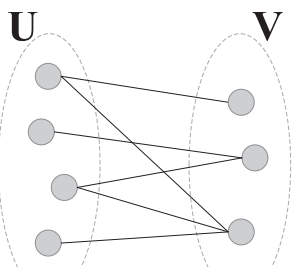

(a) (b)

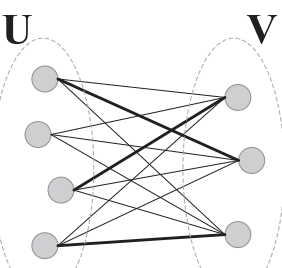

Fig. 2. An example of (a) bipartite graph and (b) complete bipartite graph.

decide one-shot from which seller he is going to buy a channel. In the other hand, it needs to obtain collectively all the bids submitted to every seller and then to compute market-clearing prices for all the sellers. It requires central control over or coordination among the sellers, which is not practical for the distributed networks (e.g., see example of double auction in [17]). Thus one-shot auction is not suitable for multiseller networks without any cooperation or centralized entity.

Considering the deficiency of one-shot auction in multiseller case, we design the spectrum auction in the distributed $\mathrm{CR}$ networks with multiple POs as a multishot auction, since it can be easily implemented in a distributed manner. We denote the proposed dynamic auction as MAP auction which will be discussed in detail in Section 5 .

\section{Optimal Channel Assignment}

In this section, we transform the problem in (4) into an optimal matching problem [20] in graph theory and derive the optimal channel assignment by means of KuhnMunkres algorithm [22], which is essentially a centralized algorithm. Although the centralized algorithm is not realistic for practical system as mentioned in Section 3.2, we present the centralized approach as a benchmark for the auction-based solution in the simulations.

\subsection{Optimal Matching Problem}

To better understand the optimal matching problem [20], we first introduce some basic concepts related with the bipartite graph and matching in graph theory.

A bipartite graph is a graph whose vertices can be divided into two disjoint sets $\mathbf{U}$ and $\mathbf{V}$ such that every edge connects a vertex in $\mathbf{U}$ to one in $\mathbf{V}$. A complete bipartite graph is a special bipartite graph where every vertex of $\mathbf{U}$ is connected to every vertex of $\mathbf{V}$. Since a complete bipartite graph is completely determined by the vertex sets $\mathbf{U}$ and $\mathbf{V}$, we can write it as $G=(\mathbf{U}, \mathbf{V})$ for simplicity. A weighted graph is such a graph wherein every edge is associated with a weight which is usually a real number. Thus a weighted complete bipartite graph can be written as $G=(\mathbf{U}, \mathbf{V}, \mathbb{W})$, where $\mathbb{W}=\left\{\omega_{i j}\right\}_{|\mathbf{U}| \times|\mathbf{V}|}$ and $\omega_{i j}$ is the weight of edge connecting with vertexes $i \in \mathbf{U}$ and $j \in \mathbf{V}$.

A matching in a bipartite graph is a set of edges without common vertices, that is, a matching is a set of edges such that for each vertex in the graph at most one edge in the matching is incident upon this vertex. Fig. 2 presents an example of the bipartite graph (Fig. 2a) and the complete

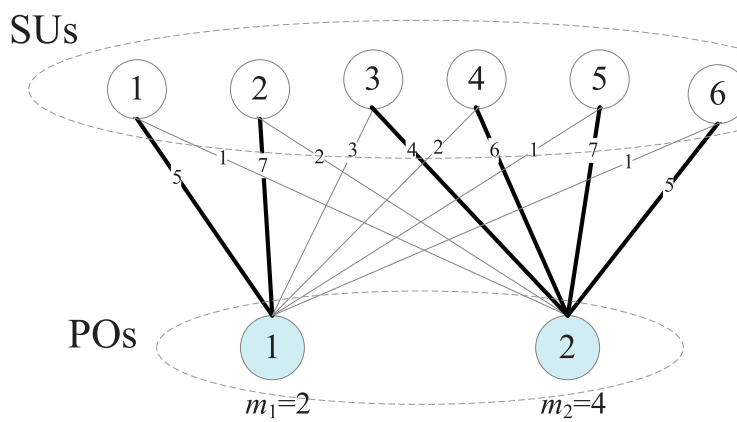

Fig. 3. A graphic representation for the system model in Fig. 1.

bipartite graph (Fig. 2b), where the bold edges in Fig. $2 b$ match the complete bipartite graph.

Now we can formally write the optimal matching problem in a weighted complete bipartite graph as follows: Given a weighted complete bipartite graph $G=(U, V, \mathbb{W})$, find a matching (a set of edges) with maximum weight.

The optimal matching problem can be solved by the Kuhn-Munkres algorithm (also known as Hungarian algorithm) in polynomial time [22]. Due to space limitation, we do not present in detail the algorithm in this work. Thus a nature thinking is to transform the original problem into an optimal matching problem.

\subsection{Optimal Channel Assignment}

It is easy to see that each CR network can be represented as a weighted complete bipartite graph $G=(\mathbf{U}, \mathbf{V}, \mathbb{W})$, where $\mathbf{U}$ is the set of SUs, $\mathbf{V}$ is the set of POs and $\mathbb{W}$ is an $N \times M$ matrix with $\omega_{n i}=v_{n i}-c_{i}, \forall n \in N, i \in M$. Obviously each channel assignment $\mathbb{R}$ corresponds to a set of edges in $G$. Fig. 3 presents a graphic representation for the system model in Fig. 1, wherein the set of bold edges is equivalent to the channel assignment in Fig. 1. Thus the original problem in (4) can be transformed into the following problem: find a set of edges with maximum weight, subjecting to $\rho_{n} \leq 1, \forall n \in N$ and $\rho_{i} \leq m_{i}, \forall i \in M .^{5}$ However, it is still not an optimal matching problem. ${ }^{6}$ This can be shown in Fig. 3 , where the optimal channel assignment (shown as the bold edges) is not a matching.

Thus it is necessary to make some skillful change to Fig. 3 so as to transform the original optimization problem into an optimal matching problem. We split the vertex corresponding to each PO $i$ as $m_{i}$ identical vertices, and split the edge between SU $n$ and PO $i$ into $m_{i}$ identical edges each connecting SU $n$ to a new generated vertex, as shown in Fig. 4. Such a new graph is referred to as the splitting graph. In fact, the splitting graph is a weighted complete bipartite graph $G=\left(\mathbf{U}, \mathbf{V}^{\dagger}, \mathbb{W}^{\dagger}\right)$, where $\mathbf{U}$ is the set of $S U s, \mathbf{V}^{\dagger}$ is the set of all residual channels and $\mathbb{W}^{\dagger}$ is a column extension of $\mathbb{W}$. We find that the original problem in (4) is exactly an optimal matching problem in the splitting graph. Thus we can use the Kuhn-Munkres algorithm [22] to derive the optimal matching. The set of bold edges in Fig. 4 is an optimal matching, which is equivalent to the channel assignment in Fig. 3.

5. The notation $\rho_{i}$ is the degree of vertex corresponding to PO $i \in M$ and $\rho_{n}$ is the degree of vertex corresponding to SU $n \in N$.

6. In fact, it is an optimal matching problem only if $m_{i}=1, \forall i \in M$. 


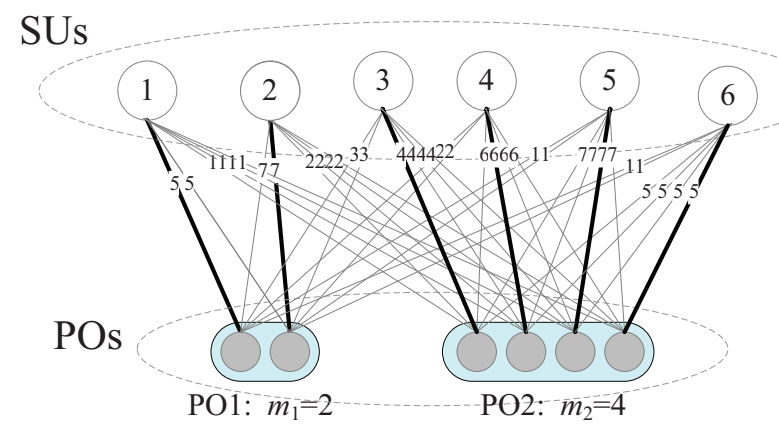

Fig. 4. A splitting graph for the system model in Fig. 1.

It is worth noting that the Kuhn-Munkres algorithm is a centralized algorithm which is unrealistic for practical system. Nevertheless, we provide the centralized approach as a benchmark for the auction-based solution in the analysis and simulations.

\section{Auction-Based Channel Assignment}

In this section, we present the basic elements and detail mechanism of MAP, and then we prove the convergence and optimality of MAP. We further analyze the incentive issues and distributed implementation of MAP protocol in practical networks.

\subsection{Basic Elements of MAP}

As mentioned previously, MAP is a kind of dynamic auction wherein the auctioneers set the trading prices and the bidders select the best auctioneer for bidding.

The strategy of each SU is to decide whether to buy a channel and from which PO he is going to buy a channel. Formally, we can write the strategy of SU $n$ as:

$$
\mathbf{x}_{n}=\left(x_{n 1}, x_{n 2}, \ldots, x_{n M}\right),
$$

where $x_{n i} \in\{0,1\}$ represents the willingness of SU $n$ to buy a channel from PO $i$, that is, $x_{n i}=1$ denotes that $\mathrm{SU} n$ is willing to buy a channel from PO $i$ and $x_{n i}=0$ otherwise. $^{7}$ Note that $\mathbf{x}_{n}=\mathbf{0}_{M}$, where $\mathbf{0}_{M}$ is an $M$-dimensional vector whose all components are zero, denotes that SU $n$ does not want to buy any channel.

Since each SU can only use one channel at a particular time, we have $\sum_{i \in M} x_{n i} \leq 1$ for all $n \in N$. Thus we can easily write the strategy space of SUs as $\Theta=\left\{\mathbf{e}_{M}^{0}, \mathbf{e}_{M}^{1}, \ldots, \mathbf{e}_{M}^{M}\right\}$ where $\mathbf{e}_{M}^{0}=\mathbf{0}_{M}$ and $\mathbf{e}_{M}^{i}, i \neq 0$, is an $M$-dimensional vector whose $i$ th component is 1 and all others 0 . Recall the example in Fig. 1, we have $\mathbf{x}_{1}=\mathbf{x}_{2}=\mathbf{e}_{2}^{1}$ and $\mathbf{x}_{3}=\mathbf{x}_{4}=\mathbf{x}_{5}=$ $\mathbf{x}_{6}=\mathbf{e}_{2}^{2}$. The strategy profile of SUs, denoted by is defined by all SUs' strategies:

$$
\mathbf{X}=\left(\begin{array}{c}
\mathbf{x}_{1} \\
\ldots \\
\mathbf{x}_{N}
\end{array}\right),
$$

where $\mathbf{x}_{n} \in \Theta$ for all $n \in N$.

From a market perspective, we define the demand for channels of PO $i$, denoted by $d_{i}$, as the number of SUs who

7. For simplicity, we will use the following statements interchangeably: 1) SU $n$ is willing to buy a channel from PO $i$, 2) SU $n$ chooses PO $i$ for bidding, 3) SU $n$ places (submits) his bid on (to) PO $i$. are willing to buy channel from PO $i$. Formally, we can write $d_{i}$ as a function of i.e.,

$$
d_{i}=\sum_{n \in N} x_{n i}, \quad \forall i \in M
$$

The supply of each PO $i$ is defined as the number of residual channels he owns, i.e., $m_{i}$. We say a PO $i$ in demanded surplus (or over demanded) if the demand for channels of PO $i$ exceeds the supply of PO $i$, i.e., $d_{i}>m_{i}$, and we say PO $i$ in supply surplus otherwise. Recall the example in Fig. 1 , we have $m_{1}=d_{1}=2$ and $m_{2}=d_{2}=4$, and thus both of PO1 and PO2 are not in demanded surplus (or supply surplus).

Since each channel can only be used by one SU at a particular time, it is necessary to design a mechanism for each $\mathrm{PO}$ to deal with the situation of demanded surplus. A simple mechanism, denoted by $\mathcal{D}^{0}$, is that each PO $i$ randomly selects $m_{i}$ SUs to which he is going to sell the channels, from the set of SUs who select the strategy $\mathbf{e}_{M}^{i}$. This mechanism may result in low profit of the PO itself, and meanwhile lower the spectrum utilization of the whole network due to the wild behavior of dropping SUs randomly. Thus a more intelligent mechanism is necessary for each PO to deal with demanded surplus.

In essence, such a mechanism is part of the strategy of the PO. In dynamic auction, the trading prices are set by auctioneers and announced to all bidders in an open manner. It is obvious that the trading price is also part of each PO's strategy. Thus the strategy of each PO $i$ can be defined as follows:

$$
\mathbf{y}_{i}=\left\{p_{i}, \mathcal{D}_{i}\right\}
$$

where $p_{i}$ is the trading price associated with $\mathrm{PO} i$ and $\mathcal{D}_{i}$ is the mechanism to deal with demanded surplus. Further, the trading price vector, denoted by $\mathbf{p}$, is defined by all POs' trading prices:

$$
\mathbf{p}=\left(p_{1}, p_{2}, \ldots, p_{M}\right)
$$

Similarly, the strategy profile of POs, denoted by $\mathrm{Y}$, is defined by all POs' strategies:

$$
\mathbb{Y}=\left(\begin{array}{c}
\mathbf{y}_{1} \\
\ldots \\
\mathbf{y}_{M}
\end{array}\right) .
$$

From an economic perspective, if $\mathrm{PO} i$ sells a residual channel to $\mathrm{SU} n$ at price $p_{i}$, the profit of $\mathrm{PO} i$ is defined as the difference between the selling price and cost of the channel, i.e., $p_{i}-c_{i}$, and the profit of $\mathrm{SU} n$ is defined as the difference between his valuation for the purchased channel and the purchasing price, i.e., $v_{n i}-p_{i}$. Additionally, if a residual channel of $\mathrm{PO} i$ is not purchased by any $\mathrm{SU}$, the profit of this channel is zero for PO $i$. Similarly, if an SU does not buy any channel from POs, his profit is zero. It is notable that the net profit of an assignment (say, from PO $i$ to $\mathrm{SU} n$ ) is exactly the aggregate profit of the associated PO and SU, i.e., $s_{n i}=p_{i}-c_{i}+v_{n i}-p_{i}$, since the selling price and purchasing price cancel each other and do not interference the social wealth.

The utility of each SU $n$, denoted by $u_{n}$, is defined as his profit under current strategy profile. In details, as SU $n$ chooses the PO $i$ for bidding, i.e., $\mathbf{x}_{n}=\mathbf{e}_{M}^{i}$, his utility is 
$v_{n i}-p_{i}$ if $\mathrm{PO} i$ accepts the request of $\mathrm{SU} n$ and 0 otherwise.

Formally, for SU $n$ with $\mathbf{x}_{n}=\mathbf{e}_{M}^{i}$, we have:

$$
u_{n}=\left\{\begin{array}{ll}
v_{n i}-p_{i}, & i \in M, \text { accepted by } \mathrm{PO} i \\
0, & i \in M, \text { refused by } \mathrm{PO} i \\
0, & i=0
\end{array} .\right.
$$

The utility of each PO $i$, denoted by $\pi_{i}$, is defined as the aggregate profit of all residual channels he owns. In details, if PO $i$ is in demanded surplus, i.e., $d_{i}>m_{i}$, he can sell out all residual channels he owns, and gain profit from all residual channels. While if PO $i$ in supply surplus, i.e., $d_{i}<m_{i}$, he can only sell $d_{i}$ residual channels, and gain profit from $d_{i}$ residual channels. Thus we can formally write $\pi_{i}$ as follows:

$$
\pi_{i}=\left\{\begin{array}{cc}
m_{i} \cdot\left(p_{i}-c_{i}\right), & d_{i} \geq m_{i} \\
d_{i} \cdot\left(p_{i}-c_{i}\right), & d_{i}<m_{i} .
\end{array}\right.
$$

So far, we have defined the essential elements of MAP, i.e., the strategies and utilities of both auctioneers and bidders. Thus MAP can be formally defined as follows:

1. Bidders/Auctioneers/Auctioned Items: the same as those in spectrum auction;

2. Strategies: St. of bidder is in (5), and St. of auctioneer is in (8);

3. Utilities: Ut. of bidder is in (11), and Ut. of auctioneer is in (12).

\subsection{Mechanism of MAP}

We first provide the essential assumptions for MAP: all POs and SUs are selfinterested which means they are always to maximize their own utility, and myopic which means they act to maximize their immediate expected utility. Thus each PO is willing to sell his channels with a price higher than the reservation price and each SU is willing to buy the channel with a price lower than his valuation for this channel. The basic idea for MAP is that: each PO systematically adjusts the trading price and each SU subsequently chooses the best PO for bidding.

In MAP, the auctioning process goes like this: from a seller's perspective, each PO starts by calling out a low trading price and then gradually raises the price as long as he is in demanded surplus. From a buyer's perspective, each SU indicates whether to buy a channel and which channel he is going to buy at the given trading price vector. As a PO's trading price rises, the SUs naturally reduce their interest for buying the channels of this PO. The auction ends as none of the $\mathrm{PO}$ is in demanded surplus and each PO sells his channels, at current trading price, to those SUs who choosing this $\mathrm{PO}$ for bidding in current trading price vector. ${ }^{8}$

Due to the progressive nature of MAP, we define MAP as a round-based distributed process which works as follows: In the first stage of each round, each PO elicits the demands for his channel in the previous round and judges whether he is in demanded surplus. If so, the PO raises his trading price by a given step and announces the new price to all SUs. Such a price updating and announcing process is referred to as Asking (of $\mathrm{PO}$ ). In the second stage of each

8. In fact, each PO $i$ chooses mechanism $\mathcal{D}^{1}$ to deal with demanded surplus, where $\mathcal{D}^{1}$ can be explained as "raise price and refuse all."
TABLE 2

Mechanism of MAP

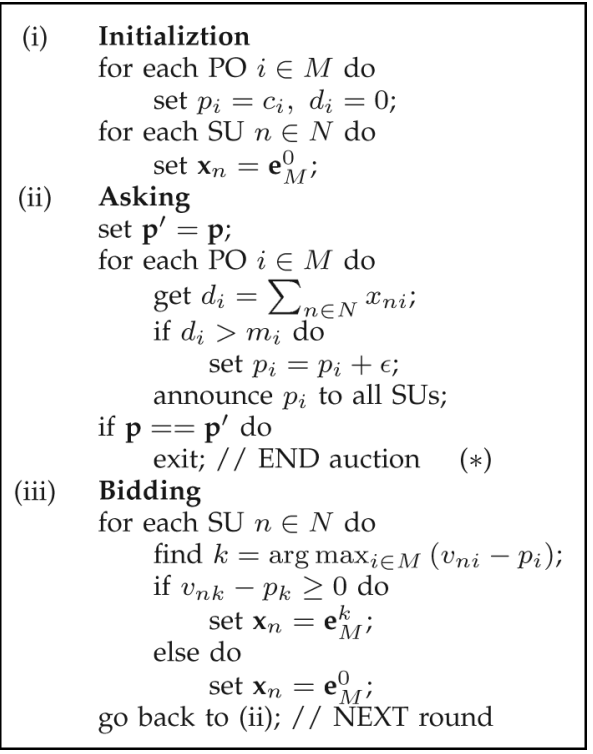

round, each SU decides whether to buy a channel, and if so, selects the PO which maximizes his utility for bidding, according to the trading prices vector POs just announced. Such a process is referred to as Bidding (of SU). The Asking/ Bidding process keeps on until none of the POs is in demanded surplus. In a word, the essence of MAP is that: by raising the trading prices of the POs in demanded surplus, the SUs who were willing to buy channels from these POs will be driven to other POs. Formally, we present the detailed mechanism of MAP in Table 2, where $\mathbf{p}^{\prime}$ is the trading price vector in previous round and step $\epsilon$ is an arbitrarily small positive number which determines the converging speed.

Remark 1. The Asking/Bidding process cannot occur infinitely because, when the trading price of a PO becomes sufficiently large, say, higher than all SUs' valuations for his channel, the PO cannot be in demanded surplus (since none of the SUs will place bid on this PO).

Remark 2. The initial price of each PO $i$ can be set higher than $c_{i}$. Let $p_{i}^{T}$ be the trading price of PO $i$ at the last round of MAP. In fact, as long as PO $i$ chooses an initial price lower than $p_{i}^{T}$, the trading price $p_{i}$ will always converges to $p_{i}^{T}$, and the achieved channel assignment is same to the primal one. However, if PO $i$ chooses an initial price higher than $p_{i}^{T}$, the achieved channel assignment may be different.

Remark 3. The strategies of SUs (i.e., $)$ in the last round of MAP is actually the resulting channel assignment. This is due to the fact that, none of the POs is in demanded surplus as the auction ends, and thus each SU who places a bid on any PO is actually assigned a channel by this PO. Equivalently speaking, if an SU $n$ is assigned a channel by any PO say $i$, then his best strategy in the last round must be $\mathbf{x}_{n}=\mathbf{e}_{M}^{i}$, and if an SU $n$ is not assigned any channel, then his best strategy in the last round must be $\mathbf{x}_{n}=\mathbf{e}_{M}^{0}$. 


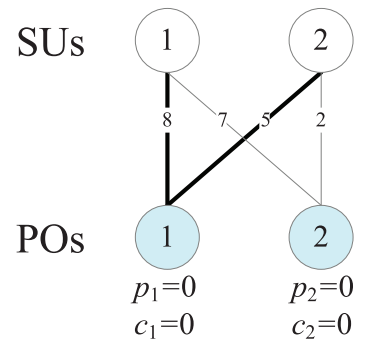

(a)

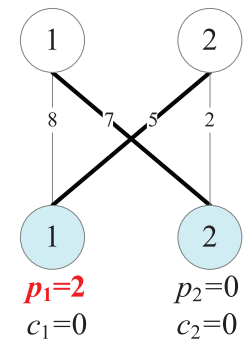

(b)
Fig. 5. An example of MAP in a system with 2 POs and 2 SUs: (a) the first round, and (b) the last round.

Fig. 5 presents an example of MAP in a system with 2 POs (each owning 1 residual channel) and 2 SUs. The bold edge indicates the bidding interest of SUs at a given trading price vector. The number on each edge denotes the valuation of SU for the associated channel. ${ }^{9}$ Without loss of generality, we assume that the reservation prices of both POs are zero, and thus each PO will calling out an initial price at zero. At this trading price vector, both SUs will place their bids on PO1, which results in demanded surplus in PO1, as shown in Fig. 5a. According to the MAP mechanism, PO1 raises his price $p_{1}$ up to $p_{1}>1$, say $p_{1}=2$, and SU1 will move his bid to PO2, as shown in Fig. 5b. Now none of the POs is in demanded surplus and thus the auction ends. The PO1 assigns the channel to SU2 and PO2 assigns the channel to SU1. It is notable that the channel assignment achieved by MAP is exactly the optimal channel assignment.

\subsection{Equilibrium of MAP}

We analyze the convergence of MAP in this section. Without loss of generality, we assume that the SUs have different valuations for the same channel, i.e., $v_{m i} \neq v_{n i}$, $\forall m \neq n$. In one hand, the SUs with different services may choose different form of valuation function to evaluate the value of channel, and thus they have different valuations. Even the SUs choose the same valuation function, this assumption is reasonable because the channel SNR are different for different SUs, as we distribute the SUs randomly in a testing area.

We first define weak equilibrium (W.E.) as a state in which none of the POs is in demanded surplus. Formally, we present the definition of W.E. as follows:

Definition 1 (Weak Equilibrium). W.E. is defined as a state in which the demand for channels of each $P O i$ does not exceed the supply of $P O$ i, i.e., $d_{i} \leq m_{i}, \forall i \in M$.

The most important property of W.E. is that each SU who submits a bid to any $\mathrm{PO}$ is actually assigned a channel by this PO, since there is no PO in demanded surplus. We find that MAP converges to W.E., and we show this property in the following lemma.

Lemma 1. MAP converges to a W.E.

Proof. We first show that the Asking/Bidding procedure in MAP cannot go on infinitely. Let $\widetilde{p}_{i}=\max _{n \in N} v_{n i}$ and

9. Note that we do not confuse with the number on the edges in Fig. 4 which denotes the net profit of an assignment.

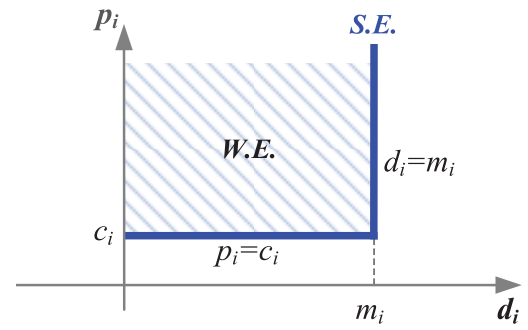

Fig. 6. Summary of W.E. and S.E. with different properties.

$\bar{p}_{i}=\widetilde{p}_{i}+\epsilon, \forall i \in M$. We can easily find that, when the trading price of PO $i$ achieves sufficiently high, say, $p_{i}>\widetilde{p}_{i}$, PO $i$ cannot be in demanded surplus and raise his price any more. Thus we have $\mathbf{p} \preceq \overline{\mathbf{p}}$ where $\overline{\mathbf{p}}=\left(\bar{p}_{1}, \bar{p}_{2}, \ldots, \bar{p}_{M}\right) \cdot{ }^{10}$ Note that in each round of MAP there is at least one PO raising his price by $\epsilon$, thus the above $\overline{\mathbf{p}}$ must be achieved, if possible, in at most $K$ rounds, where $K=\sum_{i \in M}\left(\bar{p}_{i}-c_{i}\right) / \epsilon$ is a finite number since the components of $\mathbf{c}$ and $\mathbb{W}$ are finite and the step $\epsilon$ is not infinitesimal. Thus we show that MAP ends in finite rounds. In addition, in the end of MAP (i.e., line $(*)$ in Table 2), there is no PO in demanded surplus, which is exactly a W.E. state.

It is notable that W.E. is usually inefficient. For example, when all POs set very high trading prices, say $\mathbf{p}=\overline{\mathbf{p}}$, none of the SUs will decide to buy channel and the spectrum utilization is zero, but this is yet a W.E. Due to the limitation of W.E., we introduce the concept of strong equilibrium (S.E.) or equilibrium in short.

Definition 2 (Strong Equilibrium). S.E. is defined as a state in which 1) the demand for channels of each $P O i$ does not exceed the supply of $P O$ i, i.e., $d_{i} \leq m_{i}, \forall i \in M$, and 2) if the demand for channels of $P O i$ is less than the supply of $P O i$, then the trading price of $P O$ i equals his reservation price, i.e., $p_{i}=c_{i}$ if $d_{i}<m_{i}$.

It is obvious that an S.E. is always a W.E. In fact, in S.E., each PO $i$ is either in the state of 1) $d_{i}<m_{i}$ and $p_{i}=c_{i}$ or 2) $d_{i}=m_{i}$, while in W.E., each PO $i$ is in the state of $d_{i} \leq m_{i}{ }^{11}$ We further define the weak equilibrium price and (strong) equilibrium price as the trading price vectors in W.E. and S.E., respectively.

In order to provide an intuitionistic impression, we show the summary of W.E. and S.E. by properties in Fig. 6 . The S.E. is shown as the bold lines, i.e., $p_{i}=c_{i}$ and $d_{i}=m_{i}$, and the W.E. is shown as the range surrounded by $p_{i}=c_{i}, d_{i}=$ $m_{i}$ and $p_{i}$ axis.

Fig. 7 presents an example of W.E. (price) and S.E. (price) in a system with 2 SUs and 2 POs (each owning 1 residual channel), in which Fig. 7a shows a W.E., Fig. 7b shows an S.E., and Fig. $7 b^{\prime}$ is an equivalent graph of Fig. $7 \mathrm{~b}$ by subtracting the reservation prices from the trading prices and valuations. It is easy to see that $d_{1}<m_{1}$ and $p_{1}>c_{1}$ in Fig. $7 \mathrm{a}$, thus it is not an S.E. In both Figs. $7 \mathrm{a}$ and $7 \mathrm{~b}, \mathrm{SU1}$ is going to buy a channel from PO2 while SU2 does not buy

10. If $\mathbf{x}$ and $\mathbf{y}$ are $M$-dimensional vectors, then $\mathbf{x} \preceq \mathbf{y}$ denotes that for all $i \in M, x_{i} \leq y_{i}$, and $\mathbf{x} \succeq \mathbf{y}$ otherwise.

11. Note that $p_{i}$ is impliedly greater than $c_{i}$ for each PO $i$. 
W.E.

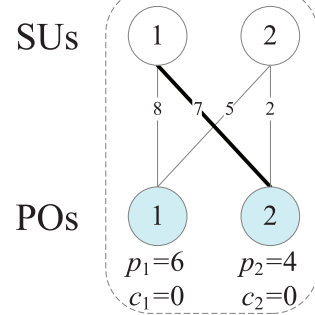

(a)
S.E.

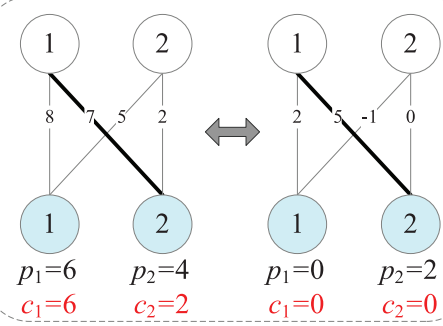

(b')
Fig. 7. An example of W.E. and S.E. in a system with 2 POs and 2 SUs.

any channel from POs, thus the resulting channel assignment is $r_{12}=1$ and $r_{21}=r_{11}=r_{22}=0$. The social income achieved by the W.E. in Fig. $7 \mathrm{a}$ and S.E. in Figs. $7 \mathrm{~b}$ or $7 \mathrm{~b}^{\prime}$ are $S=7$ and $S=5$, respectively.

Although the valuations in Figs. $7 \mathrm{a}$ and $7 \mathrm{~b}$ are same, the optimal channel assignment and optimal social income are different due to the difference of reservation prices. Specifically, in Fig. 7a, the optimal channel assignment is $r_{12}^{*}=r_{21}^{*}=1$ and $r_{11}^{*}=r_{22}^{*}=0$, and the optimal social income is $S^{*}=7+5=12$, while in Figs. $7 \mathrm{~b}$ or $7 \mathrm{~b}^{\prime}$, the optimal channel assignment is $r_{12}^{*}=1$ and $r_{21}^{*}=r_{11}^{*}=$ $r_{22}^{*}=0$, and the optimal social income is $S^{*}=7-2=5$. So we can find that the W.E. in Fig. 7a does not achieve the optimal channel assignment or optimal social income, while the S.E. in Figs. $7 \mathrm{~b}$ or $7 \mathrm{~b}^{\prime}$ does.

In what follows, we will show that, given a small enough step $\epsilon$, MAP converges to an S.E.

Lemma 2. MAP converges to an S.E., if $\epsilon$ is small enough.

Proof. We have proved that MAP converges to a W.E. in Lemma 1, i.e., $d_{i} \leq m_{i}, \forall i \in M$. Thus we can prove this lemma, as long as we show that: if $d_{i}<m_{i}$, then $p_{i}=c_{i}$, We prove the above assertion by contradiction. Assuming that MAP converges to W.E. state wherein there exists a PO $i$ with $d_{i}<m_{i}$ and $p_{i}>c_{i}$.

For simplicity, we denote $p_{i}^{k}$ as the trading price of $\mathrm{PO}$ $i$ at the $k$ th round, and obviously $p_{i}^{1}=c_{i}$ and $p_{i}^{T}=p_{i}$ where $T$ is the last round of MAP. Since $p_{i}^{T}>p_{i}^{1}$ and $p_{i}^{k}$ is monotonously nondecreasing with respect to $k$ (for all $k \in T)$, there must be a round $\tau$ such that $p_{i}^{\tau+1}>p_{i}^{\tau}$, that is, $\mathrm{PO} i$ is in demanded surplus in the $\tau$ th round. Strictly speaking, we have $p_{i}^{\tau+1}=p_{i}^{\tau}+\epsilon$. When $\epsilon$ is small enough, it is possible that only one buyer of PO $i$ changes his strategy at that moment. In fact, as long as 1) the step $\epsilon$ is small enough and 2) the SUs have different valuations for each channel, PO $i$ can always driven away at most one SU at a particular round as in demanded surplus. Thus the reduction of $d_{i}$ is at most one in a round, which implies that $d_{i}$ always approaches $m_{i}$.

As long as $d_{i}$ approaches $m_{i}, \mathrm{PO} i$ will no longer raise his trading price (until $d_{i}$ exceeds $m_{i}$ again, if possible). It is notable that if $\mathrm{PO} i$ does not change his trading price, the demand for his channels (i.e., $d_{i}$ ) will never decrease. This is due to the fact that the trading prices of other POs are nondecreasing.

So far, we have shown that: 1) if $p_{i}>c_{i}$ in the end of MAP, then there must be a round such that $\left.d_{i}>m_{i}, 2\right)$ as long as $d_{i}>m_{i}$ at any round, $d_{i}$ will decrease one by one

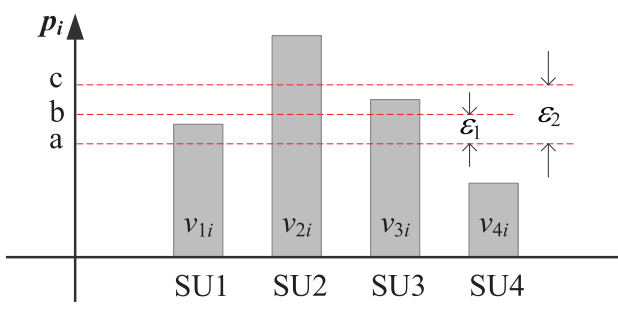

Fig. 8. An illustration of the influence of $\epsilon$ on the convergence of MAP.

and approach $m_{i}$ finally, and 3) as long as $d_{i}$ approaches $m_{i}$, it will always hold at $m_{i}$ or become greater than $m_{i}$ again. From above, we prove that: if $p_{i}>c_{i}$, then $d_{i} \geq m_{i}$, which contradicts with the assumption, and thus we complete the proof.

Remark 4. Lemma 2 holds only if $\epsilon$ is small enough. However, perfectly determining the feasible range (in particular the upper-bound) of $\epsilon$ is difficult or even sometimes impossible. In what follows, we will present a rough range of $\epsilon$. We first derive the feasible upperbound of $\epsilon$ for each PO $i$ at a particular round $t$, denoted by $\epsilon_{i}^{t}$. Without loss of generality, we assume that $\mathrm{PO} i$ is in demanded surplus and the SUs $1, \ldots, K$ choose $\mathrm{PO} i$ for bidding. For each SU $n \in K$, we define:

$$
\Lambda_{n} \triangleq\left(v_{n i}-p_{i}\right)-\left[\max _{k \neq i}\left(v_{n k}-p_{k}\right)\right]^{+},
$$

where $[x]^{+}=x$ if $x \geq 0$ and 0 if $x<0$. The first term in $\Lambda_{n}$ denotes the profit of SU $n$ if he chooses PO $i$, while the second term denotes the maximum profit SU $n$ can achieve if he does not choose PO $i$. Without loss of generality, we assume $\Lambda_{1} \leq \Lambda_{2} \ldots \leq \Lambda_{K}$. Note that if PO $i$ raises his trading price by $\epsilon>\Lambda_{n}$, SUs 1-n will change their strategies in the next round. Thus in order to make at most one SU change his strategy at round $t+1$, the upper-bound of $\epsilon$ for PO $i$ at round $t$ must be equal or less than $\Lambda_{2}$, i.e., $\epsilon_{i}^{t} \leq \Lambda_{2}$. Hence, we can obtain a conservative upper-bound for step $\epsilon$ as follows:

$$
\epsilon \leq \min _{t=1}^{T} \min _{i=1}^{M}\left\{\epsilon_{i}^{t}\right\}
$$

where $T$ is the last round of MAP. From above we find that determining the theoretical upper-bound of $\epsilon$ needs not only the private information, but also the whole course of auction, which is intractable. Therefore, in practical protocol, each $\mathrm{PO}$ can independently and adaptively set the step $\epsilon$ according to experience.

Fig. 8 shows an illustration of the influence of $\epsilon$ on the convergence of MAP in a system with $4 \mathrm{SUs}$ and one $\mathrm{PO} i$ (owning 2 residual channels). When the trading price is $p_{i}=a, \mathrm{SU} 1, \mathrm{SU} 2$ and $\mathrm{SU} 3$ are willing to buy the channel from PO $i$, which leads to a demanded surplus in PO $i$, and consequently $\mathrm{PO} i$ will raise the price by a step $\epsilon$. Note that $\Lambda_{1}=v_{1 i}-a, \Lambda_{2}=v_{2 i}-a, \Lambda_{3}=v_{3 i}-a$, and $\Lambda_{1} \leq \Lambda_{3} \leq \Lambda_{2}$. If $\epsilon>\Lambda_{3}$, say $\epsilon=\epsilon_{2}$, SU1 and SU3 are simultaneously driven away from $\mathrm{PO} i$, which results in $d_{i}<m_{i}$. While as we choose a relatively small step, say $\epsilon=\epsilon_{1}$, only SU1 is driven away, and we get the desired outcome. Therefore, there is a tradeoff between convergence and converging speed, that 
is, a small $\epsilon$ ensures better convergence but lower converging speed, and vice verse.

We refer to a CR network as demand-exceeds-supply (DES) network if 1) $N>\sum_{i \in M} m_{i}$, which means that the total demand of SUs is more than the total supply of POs, and 2) $v_{n i}-c_{i}>0, \forall i \in M, n \in N$, which means that each assignment has a positive net profit. From Lemma 2, we can find that, in a DES network, MAP converges to such an S.E. that all POs sell out their residual channels, which does coincide with common sense. We show this property in the following corollary.

Corollary 1. In DES networks, MAP converges to an S.E. with $d_{i}=m_{i}, \forall i \in M$, if $\epsilon$ is small enough.

Proof. We have proved that MAP converges to an S.E. in which each PO $i$ is either in 1) $d_{i}<m_{i}$ and $p_{i}=c_{i}$ or 2) $d_{i}=m_{i}$. We prove the corollary by contradiction. Assume that there exists a PO $i$ with $d_{i}<m_{i}$ and $p_{i}=c_{i}$ in the achieved S.E. We can easily find that there must be an SU, say $n$, who is not assigned any channel (and gains a zero utility) since $N>\sum_{i \in M} m_{i}$. As mentioned previously, the strategies of SUs in the last round of MAP is actually the resulting channel assignment, thus SU $n$ must select the strategy $\mathbf{x}_{n}=\mathbf{e}_{M}^{0}$. However, in DES networks we have $v_{n i}-p_{i}=v_{n i}-c_{i}>0$, in other word, SU $n$ has at least one strategy $\mathbf{x}_{n}=\mathbf{e}_{M}^{i}$ which is better than $\mathbf{e}_{M}^{0}$. Thus we have reached a contradiction.

\subsection{Efficiency of MAP}

We define the efficiency of MAP as the social income achieved by MAP. We analyze the efficiency of MAP in this section. Specifically, we will show whether the channel assignment achieved by MAP is optimal.

We can easily find that, in the networks with one PO $i$, the channel assignment of MAP is exactly an optimal channel assignment. In fact, in such a case, PO $i$ will raise his trading price up to the $m_{i}$ th highest valuation of SUs, and the $m_{i}$ SUs with highest valuation are willing to buy channels and actually acquire the channels from PO $i$. Note that such a channel assignment is an optimal channel assignment, and the optimal social income is $\sum_{n \in N^{\prime}}\left(v_{n i}-c_{i}\right)$, where $N^{\prime}$ is the set of SUs who successfully acquire the channels.

In what follows, we will show that in the networks with multiple POs, MAP also achieves the optimal channel assignment, as long as the step $\epsilon$ is small enough.

Theorem 1. The channel assignment of MAP is optimal, if $\epsilon$ is small enough.

Proof. We use duality theory of linear programming (specifically the primal-dual method [19]) to prove this theorem. By naturally defining the primal problem as a linear program, we consider the dual problem and then use complementary slackness to transform our optimization problem into a problem of solving a set of inequalities (constraints). A feasible solution for the primal problem is optimal, if and only if the corresponding inequalities in the dual problem are satisfied.

We perform the proof in two steps: first, we prove a feasible assignment matrix is optimal if it satisfies the complementary slackness conditions in primal-dual method, and then we show the channel assignment of MAP satisfies the complementary slackness conditions.

Step 1. Let $\alpha_{n}$ and $\beta_{i}$ be the dual variables corresponding to each SU $n$ and PO $i$, respectively, and $\theta_{n i}$ be the slacks for each assignment $(n, i)$. For convenience we define the slacks as: $\theta_{n i}=\alpha_{n}+\beta_{i}-s_{n i}$ where $s_{n i}=v_{n i}-c_{i}$ is the social profit of an assignment $(n, i)$. Note that, in the dual problem, the dual variables and slacks must be nonnegative, i.e., $\alpha_{n}, \beta_{i}, \theta_{n i} \geq 0, \forall n \in$ $N, i \in M$. Let $R$ be a feasible assignment matrix for the primal problem. By duality, $R$ has a maximal social income if, for every $\mathrm{SU} n \in N$ and $\mathrm{PO} i \in M$, the following conditions hold:

c.1. $\alpha_{n} \geq 0, \beta_{i} \geq 0, \theta_{n i} \geq 0$;

c.2. $\theta_{n i}=0$, if $(n, i)$ is an assignment in $R$ (i.e., $r_{n i}=1$ );

c.3. $\alpha_{n}=0$, if SU $n$ is not assigned in $R$, and $\beta_{i}=0$, if PO $i$ is not demanded in full, i.e., $d_{i}<m_{i}$.

The conditions in c.1-3 are referred to as the complementary slackness conditions in primal-dual method. The sufficiency of c.1-3 for optimality can be proved as follows L: Let $R^{\prime}=\left\{r_{n i}^{\prime}\right\}_{N \times M}$ be any feasible assignment matrix. On one hand, we can easily write the social income of $R^{\prime}$, denoted by $S\left(R^{\prime}\right)$, as follows:

$$
\begin{aligned}
S\left(R^{\prime}\right) & =\sum_{n \in N} \sum_{i \in M}\left(r_{n i}^{\prime} \cdot s_{n i}\right) \\
& =\sum_{n \in N} \sum_{i \in M}\left(r_{n i}^{\prime} \cdot\left(\alpha_{n}+\beta_{i}-\theta_{n i}\right)\right) \\
& \leq \sum_{n \in N} \sum_{i \in M}\left(r_{n i}^{\prime} \cdot\left(\alpha_{n}+\beta_{i}\right)\right) \\
& \leq \sum_{n \in N} \alpha_{n}+\sum_{i \in M} m_{i} \beta_{i} .
\end{aligned}
$$

The second line comes from the definition of $\theta_{n i}$, the third line follows because $\theta_{n i} \geq 0$ as specified in c.1, and the last line follows because $\sum_{i \in M} r_{n i}^{\prime} \leq 1$ and $\sum_{n \in N} r_{n i}^{\prime} \leq m_{i}$ (since $R^{\prime}$ is a feasible assignment matrix).

On the other hand, we can write the social income of $R$, denoted by $S(R)$, as follows:

$$
\begin{aligned}
S(R) & =\sum_{n \in N} \sum_{i \in M}\left(r_{n i} \cdot s_{n i}\right) \\
& =\sum_{n \in N} \sum_{i \in M}\left(r_{n i} \cdot\left(\alpha_{n}+\beta_{i}\right)\right) \\
& =\sum_{n \in N} \alpha_{n}+\sum_{i \in M} m_{i} \beta_{i} .
\end{aligned}
$$

The second line follows because $\theta_{n i}=0$ if $r_{n i}=1$, as specified in c.2, and the last line follows because $\alpha_{n}=0$ if $\sum_{i \in M} r_{n i}<1$ and $\beta_{i}=0$ if $\sum_{n \in N} r_{n i}<m_{i}$, as specified in c.3.

From (15) and (16), we can easily find that, if $R$ is a feasible assignment matrix which satisfies the complementary slackness conditions (c.1-3), then $S(R) \geq S\left(R^{\prime}\right)$ for arbitrary feasible assignment matrix $R^{\prime}$, which means $R$ is a channel assignment with maximum social income (or optimal channel assignment).

Step 2. Now we will show that the channel assignment of MAP satisfies the complementary slackness conditions. Let $R$ be any channel assignment achieved by MAP. If we can find a set of dual variables such that $R$ 
satisfies c.1-3, we complete the proof. To achieve this, we need to identify the dual variables for POs and SUs.

We set the dual variables according to the following rules: for SUs,

$$
\alpha_{n}=\left[\max _{i \in M}\left(v_{n i}-p_{i}\right)\right]^{+}, \quad \forall n \in N,
$$

and for POs,

$$
\beta_{i}=p_{i}-c_{i}, \quad \forall i \in M,
$$

where $[x]^{+}=x$ if $x \geq 0$ and $[x]^{+}=0$ if $x<0$. In fact, $\alpha_{n}$ in (17) is the profit of SU $n$ and $\beta_{i}$ in (18) is the unit profit of PO $i$, i.e., the profit of one channel. For simplicity, we define $\Delta_{n}=\max _{i \in M}\left(v_{n i}-p_{i}\right)$. Thus the slacks for each assignment $(n, i)$ can be written as:

$$
\begin{aligned}
\theta_{n i} & =\left[\Delta_{n}\right]^{+}+p_{i}-c_{i}-\left(v_{n i}-c_{i}\right) \\
& =\left[\Delta_{n}\right]^{+}-\left(v_{n i}-p_{i}\right) .
\end{aligned}
$$

We first prove the validity of condition c.1. It is easy to see that $\alpha_{n} \geq 0$ and $\beta_{i} \geq 0, \forall n \in N, i \in M$. As for the slacks, if $\Delta_{n} \geq 0$, we have $\theta_{n i}=\Delta_{n}-\left(v_{n i}-p_{i}\right) \geq 0$, and if $\Delta_{n}<0$, which means $v_{n i}-p_{i}<0$ for all $i \in M$, we have $\theta_{n i}=0-\left(p_{i}-v_{n i}\right)>0$.

Then we prove the validity of condition c.2. As mentioned previously, the strategies of SUs in the last round of MAP is actually the resulting channel assignment. Thus if $(n, i)$ is an assignment in $R$, the best strategy for SU $n$ must be $\mathbf{e}_{M}^{i}$ at the last round, which means $v_{n i}-p_{i} \geq 0$ and $v_{n i}-p_{i} \geq v_{n i^{\prime}}-p_{i^{\prime}}$ for all $i^{\prime} \in M$, or, equivalently, $\Delta_{n}=\max _{i^{\prime} \in M}\left(v_{n i^{\prime}}-p_{i^{\prime}}\right)=v_{n i}-p_{i} \geq 0$. Substitute the above $\Delta_{n}$ from (19), we have: $\theta_{n i}=v_{n i}-$ $p_{i}-\left(v_{n i}-p_{i}\right)=0$.

Now prove the validity of condition c.3. On one hand, if $\mathrm{SU} n$ is not assigned in $R$, then the best strategy of $\mathrm{SU} n$ must be $\mathbf{e}_{M}^{0}$ at the last round of MAP, which means $v_{n i}-$ $p_{i}<0$ for all $i \in M$, otherwise SU $n$ will choose a PO with positive profit for bidding. Thus we have $\Delta_{n}<0$ and $\alpha_{n}=\left[\Delta_{n}\right]^{+}=0$. On the other hand, if $\mathrm{PO} i$ is not demanded in full, i.e., $d_{i}<m_{i}$, then we have $p_{i}=c_{i}$ according to Lemma 2 (when $\epsilon$ is small enough). Thus we have $\beta_{i}=p_{i}-c_{i}=0$.

So far, we have proved that as we use the dual variable defined in (17) and (18), all of the complementary slackness conditions are satisfied. Thus, from Step 1 and Step 2, we can complete the proof straightforward.

To better understand the relation between dual variables (or slacks) and optimality, we recall several examples mentioned previously:

Example 1. Consider the case in Fig. 5b, which is an optimal channel assignment. By (17) and (18), we can find the following dual variables: $\alpha_{1}=\max \{6,7\}=7, \alpha_{2}=$ $\max \{3,2\}=3, \beta_{1}=2, \beta_{2}=0$. By (19), we have the following slacks: $\theta_{11}=1, \theta_{12}=0, \theta_{21}=0, \theta_{22}=1$. Obviously the conditions c.1-3 are all satisfied.

Example 2. Consider the case in Fig. 6a, it is not an optimal channel assignment. Similarly, the dual variables are: $\alpha_{1}=$ $\max \{2,3\}=3, \alpha_{2}=[\max \{-1,-2\}]^{+}=0, \beta_{1}=6, \beta_{2}=4$.
The slacks are: $\theta_{11}=1, \theta_{12}=0, \theta_{21}=1, \theta_{22}=2$. The condition c. 3 is not satisfied because $\beta_{1}=6>0$ while PO1 is not demanded in full.

Example 3. Consider the case in Fig. 6b, which is an optimal channel assignment. Similarly, the dual variables are: $\alpha_{1}=$ $\max \{2,3\}=3, \alpha_{2}=[\max \{-1,-2\}]^{+}=0, \beta_{1}=0, \beta_{2}=2$. The slacks are: $\theta_{11}=1, \theta_{12}=0, \theta_{21}=1, \theta_{22}=2$. Obviously the conditions c.1-3 are all satisfied.

\subsection{Incentive of MAP}

In this section, we analyze the incentive issues of MAP including the incentive for both POs and SUs joining the auction and the incentive for accepting the channel assignment achieved by MAP. The former incentive is regarded as individual rationality (IR), which means all of the participators can obtain nonnegative profit as joining the auction, and the later incentive is regarded as incentive compatibility (IC), which means all of the participators can achieve maximal profit by following the mechanism of the auction.

As mentioned previously, spectrum auction is actually a combination of channel assignment process and profit transfer process. From economic perspective, the former process is used to capture the social income by assigning the sellers' items to the buyers who are most desired for the items, and the latter process is used to divide the achieved social income among the buyers and sellers. In one hand, a rational channel assignment mechanism is essential for spectrum auction since the maximum achieved profit of POs (or SUs) relies on the social income. The social income is, in fact, the aggregate profits of POs and SUs, because the selling price and purchasing price cancel each other from a social perspective. In the other hand, a rational profit transfer mechanism is also essential since the actual achieved profit of POs and SUs relies to a great extent on the profit division. Obviously that if the POs extract most of the profit from the social income by charging the SUs high prices, the SUs are not likely to accept the auction result, and vice versa.

We first consider the incentive for POs and SUs joining the proposed auction MAP (i.e., IR). According to the mechanism of MAP in Table 2, each SU will select a PO with maximal and nonnegative profit for bidding, and each PO will ask a price higher than its cost. Thus both SUs and POs can achieve nonnegative profit. Further, from the social aspect, we can see from Theorem 1 that SUs and POs achieve the maximal aggregate profit.

Then we consider the incentive for both POs and SUs accepting the auction result achieved by MAP (i.e., IC). In this issue, the profit transfer process plays an essential role. The source of profit is the channel utilization of SUs, and a certain part of profit can be transferred to the associated POs by means of pricing. A straightforward pricing mechanism is the trading price solution, in which each PO $i$ charges the related SUs his trading price $p_{i}$. In such a pricing mechanism, each SU $n$ will naturally accept the result of MAP, since in each round, he chooses the strategy which maximizes his own profit, i.e., $\mathbf{x}_{n}=\mathbf{e}_{M}^{k}$ where $k=\arg \max _{i \in M}\left(v_{n i}-p_{i}\right)$. However, the profit of POs are strongly influenced by the information the POs know and the volume of demand. Specifically, the POs with more 
information about SUs or other POs can gain more profit [24], [25], [26]. Further, the POs with higher demand requirement appears to gain more profit than those with lower demand requirement, which does coincide with common sense. Note that the demand requirement of any $\mathrm{PO}$ is related with the number and location of SUs, which usually vary randomly in time. Thus in long term, the average demand requirement of any $\mathrm{PO}$ will tend to a steady amount, say $E[N] / M^{12}$ supposing all parameters of POs are same. Thus for the PO $i$ with $m_{i}<E[N] / M$, he is more willing to accept the auction result, compared to the $\mathrm{PO} i$ with $m_{i}>E[N] / M$.

From above we can find that, using trading price solution, the profit of POs and SUs vary with the change of supply-demand relation. To make the profit division more stable and fair, we introduce another pricing mechanism: Nash bargaining solution [23]. A Nash bargaining solution is a (Pareto efficient) solution to a Nash bargaining game which usually used to model bargaining interactions between one buyer and one seller. According to Nash bargaining solution, for each channel assignment $(n, i)$, the PO $i$ charges SU $n$ a price $p_{i}=v_{n i} / 2+c_{i} / 2$, thus the PO $i$ and SU $n$ gain the same profit (i.e., $v_{n i} / 2-c_{i} / 2$ ) in an assignment. In such a pricing mechanism, both POs and SUs are willing to accept the result of MAP, since POs and SUs gain half of the total profit (social income) which is maximized by the result of MAP. Note that in this case, the trading price in the auction is just a virtual price used to ensure the normal operation of the auction, while has no relation to the profit transfer.

Besides the incentive issues mentioned above, we also explore an interesting issue about interest compatibility. The concept of interest compatibility is similar to that of incentive compatibility. The latter one is widely used in mechanism design and to ensure all of the participants truthfully reveal their private information to the mechanism. However in our auction design, the participants have no need to exchange their private information, and thus the concept of incentive compatible is no long a challenge for our auction design. In our model, each SU has different preference for different POs, due to the difference on transmission techniques, channel parameters and qualities, etc. An auction is said to be interest compatible if all of the SUs will truthfully reveal their preference in bidding processes, so that the SUs can be directed to their interested POs.

\subsection{Implementation of MAP}

In this section, we discuss the implementation of MAP protocol in practical networks. Specifically, we will show how MAP can be applied in a distributed manner.

A rough sketch for the construction of MAP protocol is shown in Fig. 9, from which we can find a whole auction duration consists of an auctioning course (denoted as Auc.), in which the SUs compete for channels, and a following serving course (denoted as Serving), in which the winning SUs access the assigned channels. Let $T_{e}$ be the time interval for two consecutive auctions, which includes a span of time $T_{a}$ for auctioning course, and a span of time $T_{s}$ for serving course. Every auctioning course is divided into multiple

12. $E[x]$ denotes the expectation of $x$.

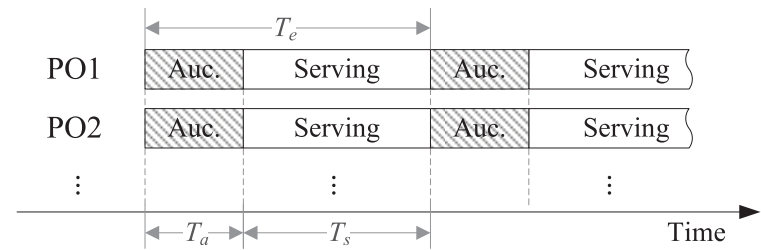

Fig. 9. The construction of MAP protocol in time dimensionality.

slots with length $T_{r}$, each corresponding to one round in auction. All network parameters (including the number of residual channels, the number and location of SUs, the SUs' requirements and valuations for channels, etc.) are assumed to be constant in one auction duration $\left(T_{e}\right)$.

To apply MAP in practical networks in a distributed manner, the following issues must be considered.

First, we assume that all POs are strictly synchronous, which means the POs start not only the auctioning course and serving course, but also each round in the auction, simultaneously. The synchronization ensures that the Asking/Bidding process in MAP proceeds in an orderly and regular fashion. Note that the synchronizing of auctioning course and serving course can be (relatively) easily achieved, since the duration of auctioning course $\left(T_{a}\right)$ and serving course $\left(T_{s}\right)$ is in large time-scale (typically on the order of seconds), but the synchronizing of round is hardly to be achieved, since the duration of round $\left(T_{r}\right)$ is usually in millisecond level. Thus we also consider the case of coarse synchronization, wherein the POs initiate each round independently. We show that the performance degradation caused by the loss of round synchronization is very limited.

Second, we assume that there exists a common control channel used for the interacting of auction signal, including the initial information, trading price and bidding. Specifically, at beginning of the auction, each PO $i$ broadcasts the initial information including channel bandwidth, carrier frequency, initial trading price, etc. In every round, each PO $i$ updates and broadcasts his trading price $p_{i}$, and then each SU $n$ decodes the trading prices and sends (unicasts) his accessing request (bidding) to the preferred PO. Note that each $\mathrm{PO}$ can also decode the trading price of others since the trading prices are broadcasted in an open manner, and thus each PO can obtain the current and previous trading price vectors so as to judge whether the auction ends.

Third, the SUs need not to sense and decode all of the trading prices. On one hand, an SU may not be able to decode the trading price of any $\mathrm{PO}$ because of the far distance between them or incompatible transmission modes they used. On the other hand, the SU may not be willing to decode the trading price of any PO (even if he can do this) for the sake of energy saving or other reasons. The SUs can simply set the unknown trading prices as infinite to prevent themselves from selecting the associated POs. In the former case, the auction result is still optimal, ${ }^{13}$ while in the latter case, the auction result may be not optimal. In fact, if each SU selects the only closest PO for sensing, the auction degenerates to a traditional fixed allocation scheme. We

13. This is due to the fact that the SU cannot use this PO's channel at all, and thus he will not choose this PO even if he knows this price. 

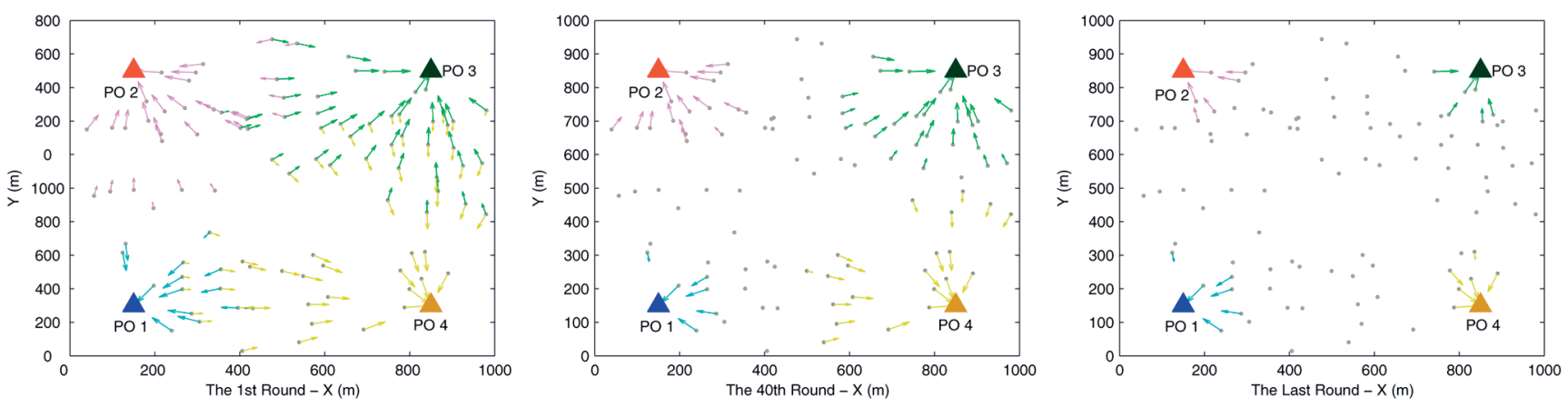

Fig. 10. Evolution of SUs' profits and preferences, using $M=4, N=100, m_{i}=6$ and fixed step $\epsilon=10$.

find that if each SU selects two closest POs for sensing, the performance degradation is very limited.

Fourth, if the auction ends within the period of auctioning course, the remanent period of auctioning course is immediately turned into serving course. While if the auction has not achieved the equilibrium at the end of auctioning course, it will be forced to terminate to guarantee a long enough serving time. In such case, there will be some POs in demanded surplus yet, and each PO $i$, if in demanded surplus, randomly selects $m_{i}$ SUs for serving. The auction result in this case is not optimal and the performance degradation may be considerable. Thus it is essential to ensure that the auction ends within the auctioning course. To achieve this, one can enlarge the period of auctioning course $\left(T_{a}\right)$, which will reduce the spectrum efficiency, or raise the step $\epsilon$, which will influence the convergence of the auction.

Fifth, the POs can set the step $\epsilon$ independently according to experience. As mentioned previously, how to determine a rational step $\epsilon$ is essential for the auction. A large step $\epsilon$ will influence the convergence of the auction and the optimality of the auction result, while a small $\epsilon$ will prolong the auctioning time and reduce the spectrum efficiency. Although (14) presents a theoretical upper-bound for $\epsilon$, it is intractable in practice. In practical protocol, each PO $i$ can adaptively adjust his step $\epsilon$ based on 1) the number of excess demand, which is defined as the difference of the demand requirement for $\mathrm{PO} i$ and the supply of $\mathrm{PO} i$ (i.e., $d_{i}-m_{i}$ ), and 2) the probability of overshooting, which is defined as the ratio of the rounds in which PO $i$ drives away two or more SUs to the whole rounds. Specifically, if the number of excess demand increases, or the probability of overshooting decreases, $\mathrm{PO} i$ can correspondingly raise his step, and vice verse. In fact, in the adaptive step scheme, we adopt an appropriate step $\epsilon$ to guarantee the probability of convergence, rather than to make the auction converges to equilibrium every time.

\section{Simulation Results}

In order to evaluate the performance of the proposed auction mechanism, we perform simulations for different network scenarios in MATLAB. Without additional indication, we will use the following configuration in all simulations: 100 SUs and 4 POs (each owning 6 residual channels) are randomly and uniformly distributed in a square area of $1000 \times 1000 \mathrm{~m}$. The carrier frequency is set to be $2 \mathrm{GHz}$ for all POs, while the channel bandwidth is set to be $\{0.5,1,1.5,2\} \mathrm{MHz}$ respectively for PO1-PO4.
The propagation path loss model is given by Friis free space equation [1], i.e., $\Gamma_{n i}=1 /\left(f_{i}^{2} d_{n i}^{2} \sigma^{2}\right)$, where $\sigma^{2}$ is the noise variance and $d_{n i}$ is the distance between PO $i$ and SU $n$ in meters. ${ }^{14}$ The noise variance is set to be $\sigma^{2}=10^{-5}$. The valuation function is modeled as the Shannon capacity (assuming data service), i.e.,

$$
v_{n i}=\mathcal{G}_{n}\left(\Gamma_{n i}, w_{i}\right)=w_{i} \log \left(1+\frac{1}{f_{i}^{2} d_{n i}^{2} \sigma^{2}}\right) .
$$

The duration of a whole auction is set to be $T_{e}=5 \mathrm{~s}$, which corresponds roughly to the time that the network environment keeps approximately constant. The length of one round is $T_{r}=10 \mathrm{~ms}$, which corresponds to the time needed for all POs and SUs to transmit one MAC layer packet (for trading price and bidding). The duration of auctioning course can be $T_{a}=\{0.1,0.2, \ldots, 5\}$ s according to different networks configurations, and accordingly the least serving time in each auction duration is guaranteed to be $T_{s}=\{4.9,4.8, \ldots, 0\} \mathrm{s}$. We run each simulation for 1,000 auction periods, which corresponds to 5,000 s according to the above setting.

\subsection{Dynamic of Auction}

To provide an intuitionistic impression for the proposed $\mathrm{MAP}$, we first present the detailed dynamic of the profits and strategies of both POs and SUs in the auction.

Fig. 10 presents an illustration of the evolution of MAP from the aspects of SUs' profits and preferences, where a fixed step $\epsilon=10$ is adopted by all POs. Each arrow denotes the profit of the incident $\mathrm{SU}$ as choosing the indicated PO, from which we can easily find the preference of the SU. The three subfigures show the profits of SUs in the first round, the 40 th round and the last round, respectively. We can find that the SUs' profits (and preferences) gradually decrease as the auction progresses, due to the increase of the $\mathrm{POs}^{\prime}$ trading prices. In particular, in the last round of $\mathrm{MAP}$, for each $\mathrm{PO}$, there are only $6 \mathrm{SUs}$ who have positive profits on this $\mathrm{PO}$ and are willing to choose this PO.

Fig. 11 presents the dynamic of the strategies of both POs and SUs using fixed step $\epsilon=10$. The left subfigure shows the dynamic of the trading prices of POs, from which we can find that the POs continue to raise their prices until the auction achieves an equilibrium state (about the 120th

14. Note that the transmitting power and transmitter/receiver antenna gain are normalized to one unit. This simplicity does not influence our results. 

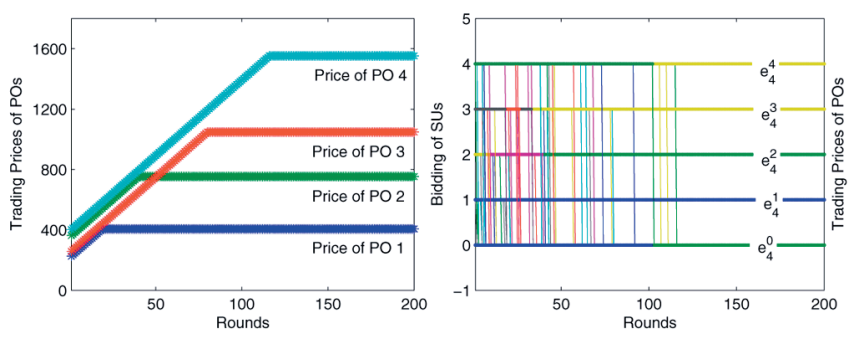

Fig. 11. Dynamic of the trading prices and bidding, using $M=4, N=100$, $m_{i}=6$ and fixed step $\epsilon=10$.

round in this example). The POs achieve different trading prices in the equilibrium. This is caused by the different bandwidth of the channels POs owning. In particular, the PO with higher bandwidth can finally address a higher trading price, and achieve higher profit in each channel, which coincides with common sense. The right subfigure shows the dynamic of the bidding of SUs, from which we find that the SUs change their bidding frequently at the beginning of MAP, and as the auction progresses, each SU's bidding strategy gradually converges to a particular one.

As can be seen from Fig. 11, using fixed step, each $\mathrm{PO} i$ raises his price in a constant speed $\epsilon$, no matter how many excess demand (i.e., $d_{i}-m_{i}$ ) he experiences. In fact, as any PO is in highly demand surplus $\left(d_{i} \gg m_{i}\right)$, he can naturally adopt a large step to increase the converging speed, while as in lowly demand surplus, he can adopt a small step to guarantee the convergence. As mentioned previously, each PO $i$ can adaptively adjust his step $\epsilon$ based on the number of excess demand and/or the probability of overshooting. In our simulations, we adopt a simple adaptive step based on the excess demand: for each $\mathrm{PO} i$, the temporal step in each round is given by:

$$
\epsilon=\tilde{\epsilon} \cdot \log _{2}\left(1+d_{i}-m_{i}\right),
$$

where $\tilde{\epsilon}$ is the baseline of adaptive step.

Fig. 12 presents the dynamic of the strategies of both POs and SUs using adaptive step $\widetilde{\epsilon}=10$. We can see that, the auction using adaptive step converges to the equilibrium (about 45 th round) much faster than that using fixed step, and the equilibrium prices achieved by two schemes are the same, which means the adaptive scheme increases the converging speed and meanwhile effectively guarantees the convergence.

\subsection{Converging Speed}

We define converging rounds, denoted by $T$, as the number of rounds MAP used to converge to the equilibrium state.
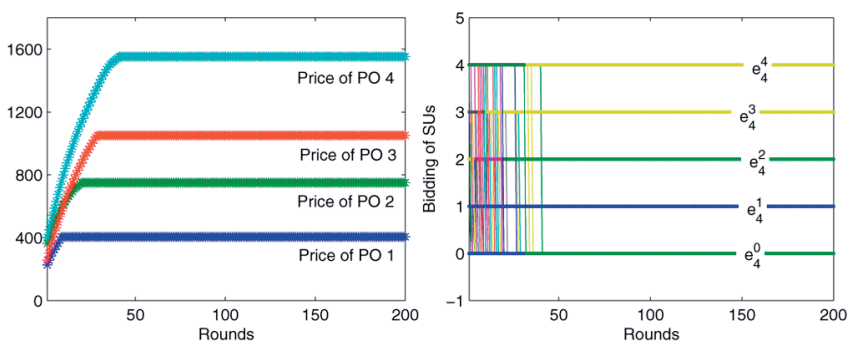

Fig. 12. Dynamic of the trading prices and bidding, using $M=4, N=100$, $m_{i}=6$ and adaptive step $\widetilde{\epsilon}=10$.

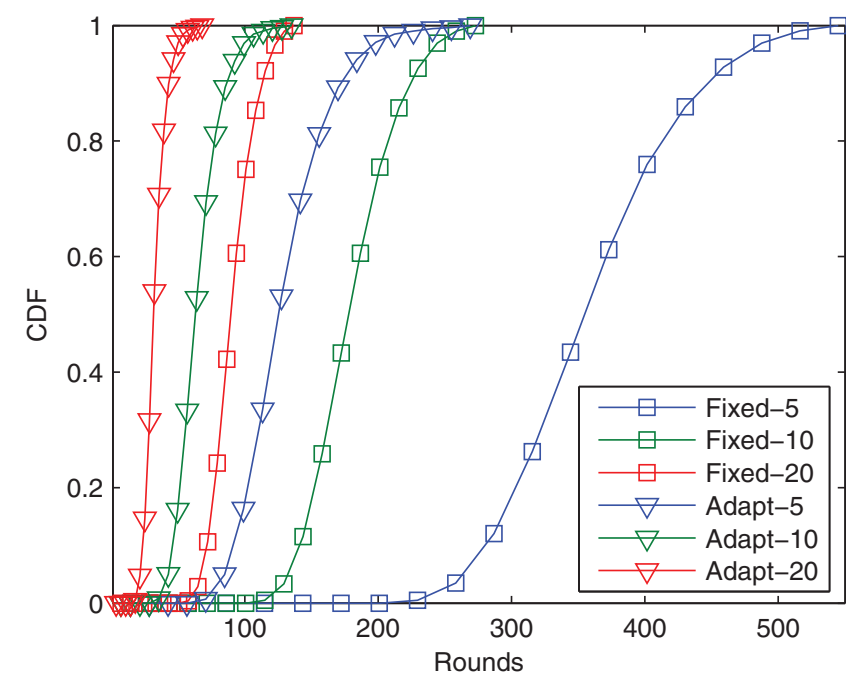

Fig. 13. CDF of converging rounds $T$, using $M=4, N=100, m_{i}=6$.

Obviously, the converging speed is straightforwardly related to the converging rounds. We show the statistic characteristics of $T$ in this section.

Fig. 13 presents the cumulative distribution functions $(C D F)$ of the converging rounds $(T)$ in different step schemes and step sizes. We can see from Fig. 13 that $T$ decreases with the increasing of step size $\epsilon$. For example, the probability of $T \leq 200$ is 0,80 , and 100 percent respectively for fixed steps $\epsilon=\{5,10,20\}$. We can further see that, for the same step size, the converging rounds in adaptive step schemes is much smaller than those in fixed stepw schemes. For example, the probability of $T \leq 200$ is 0 and 95 percent respectively for fixed step $\epsilon=5$ and adaptive step $\widetilde{\epsilon}=5$.

Fig. 14 presents the expectation of the converging rounds $(T)$ versus different step sizes $\epsilon$, from which we can also see that $T$ decreases with the increasing of step size $\epsilon$, and moreover, $T$ and $\epsilon$ are to some extent in inverse proportion. We can also see that using adaptive step schemes, $T$ can be reduced to about 30-50 percent of those using fixed step schemes with the same size.

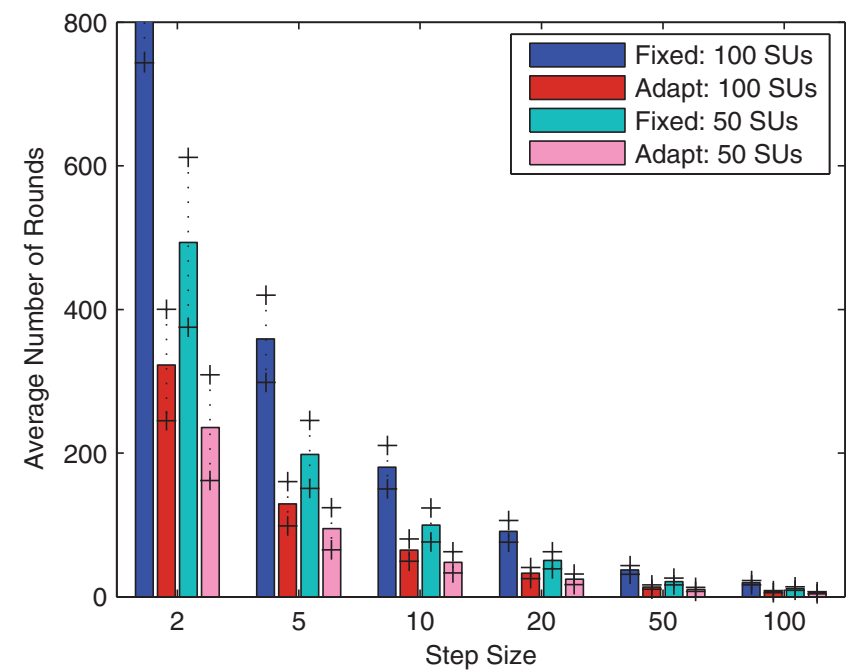

Fig. 14. Expectation of $T$ versus step $\epsilon$, using $M=4, m_{i}=6$, $N=\{50,100\}$. 


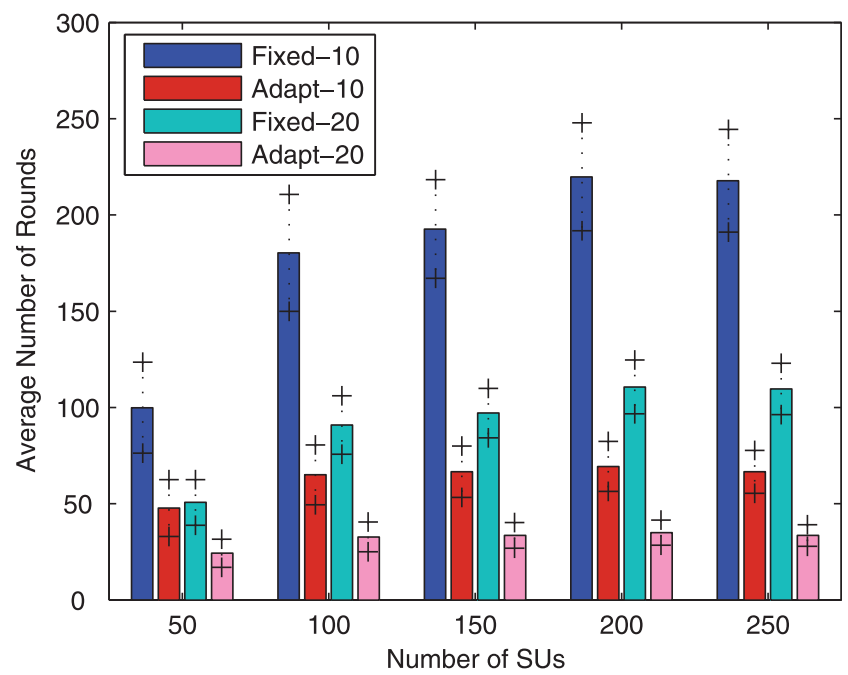

Fig. 15. Expectation of $T$ versus $N$, using $M=4, m_{i}=6, \epsilon=\{10,20\}$.

Fig. 15 presents the expectation of the converging rounds $(T)$ versus different number of SUs $(N)$, from which we can see that using fixed step schemes, $T$ increases rapidly with the increasing of the number of SUs, while using adaptive step schemes, $T$ increases very slowly as the number of SUs increases. For example, using fixed step $\epsilon=5, T$ increases from 100 to 200 as $N$ varies from 50 to 250, while using adaptive step $\widetilde{\epsilon}=5, T$ only increases from 50 to 60 . We can also see that as the number of SUs becomes greater, $T$ converges to any steady value. Thus MAP is scalable to the number of SUs, and can be applied in the network with a large number of SUs.

\subsection{Convergence and Efficiency}

MAP may converge to a W.E. or an S.E. depending on the network configurations, and note that W.E. is usually inefficiency. In this section, we investigate the influence of step scheme and step size on the equilibrium states MAP achieved. We define the convergence probability (or convergence for short) as the ratio of the number of times MAP converging to an S.E. to the total number of simulations, and the loss probability otherwise.

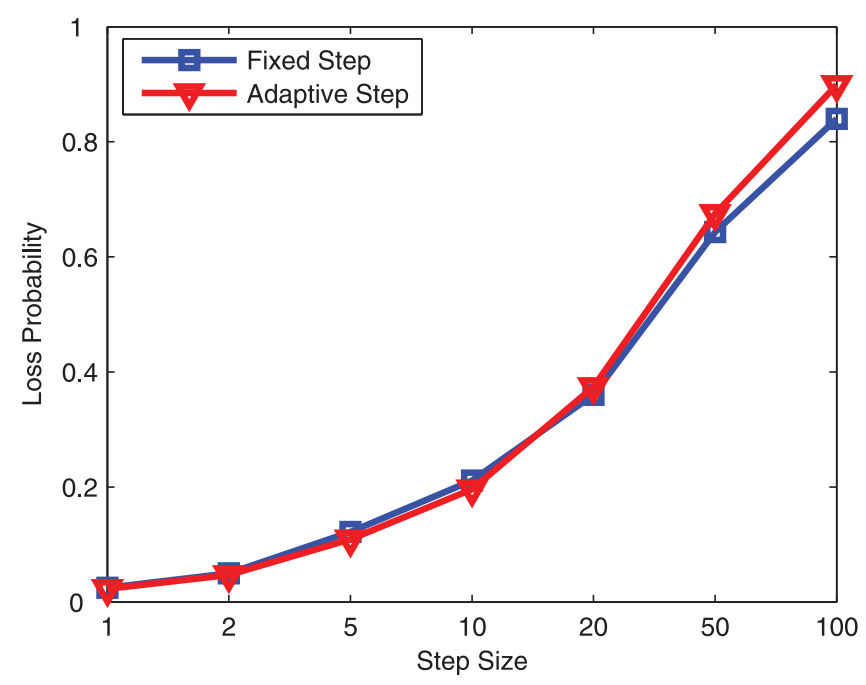

Fig. 16. Loss probability versus step $\epsilon$, using $M=4, N=100, m_{i}=6$.

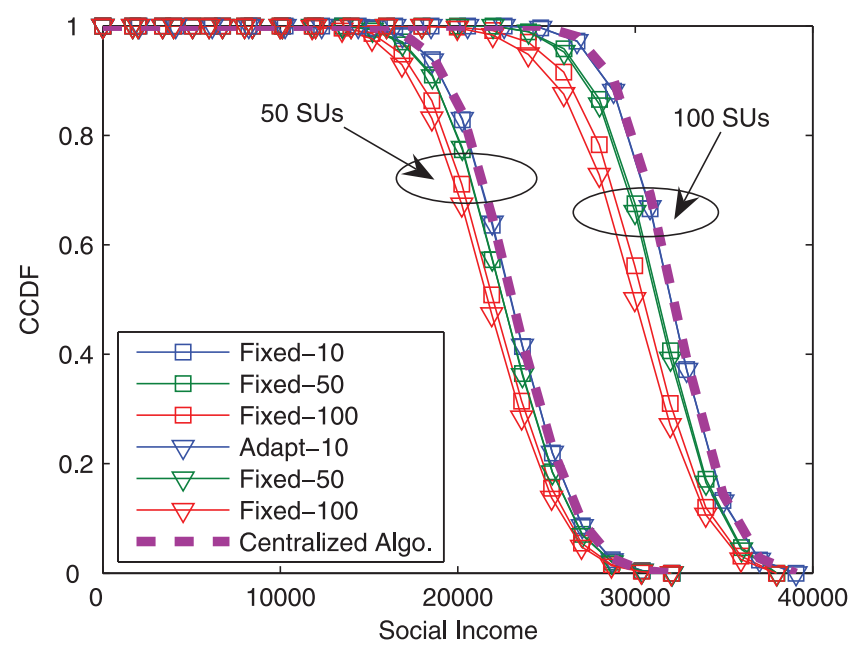

Fig. 17. CCDF of $S$ and $S^{*}$, using $M=4, m_{i}=6, N=\{50,100\}$.

Fig. 16 presents the loss probabilities in different step schemes and step sizes, from which we can see that the loss probability increases rapidly as the step size increases, and loss probability in adaptive step schemes is slightly higher than that in fixed step scheme with the same step size. Note that, although the loss probability substantially degrades as the step size increases, the performance in terms of social income (efficiency) is kept on a considerable level, as can be seen in follows.

Fig. 17 presents the complementary cumulative distribution functions (CCDF) of the optimal social income and the achieved social income $(S)$ in different step schemes and step sizes. The dashed curve denotes the optimal social income $\left(S^{*}\right)$ achieved by centralized algorithms, which actually forms the theoretical upper-bound of the achieved social income. From Fig. 17 we can see that the social incomes achieved by MAP are very close to the optimal one. For example, in the case of 50 SUs, the probability of $S \geq$ 20,000 is $85,84,80$ and 70 percent respectively for the centralized algorithm and for the proposed MAP with fixed steps $\epsilon=\{10,50,100\}$.

To provide a quantitative illustration of the degradation of social income, we present the ratio of the achieved social income $(S)$ to the optimal social income $\left(S^{*}\right)$ in Fig. 18, from which we can see that $S$ decreases slightly with the increasing of step size $\epsilon$. Such a decrease is inappreciable (less than 1 percent) in the case of $\epsilon \leq 10$, and even in the case of $\epsilon=100$, the performance degradation is less than 8 percent. Thus we can find that MAP achieve an approximately optimal channel assignment within tolerable iteration rounds.

\subsection{Equilibrium Price and Profit Transfer}

Profit transfer is essential since the actual achieved profit of POs and SUs relies to a great extent on the profit division. In this section, we investigate the equilibrium price and the profit division among POs and SUs. Since the difference of social income in fixed step and adaptive step schemes is not distinct, we only perform the simulations in fixed step schemes in this section.

Fig. 19 presents the CCDF of the equilibrium prices (of POs) in different number of SUs using fixed step $\epsilon=10$, from which we can see that the equilibrium prices increases 


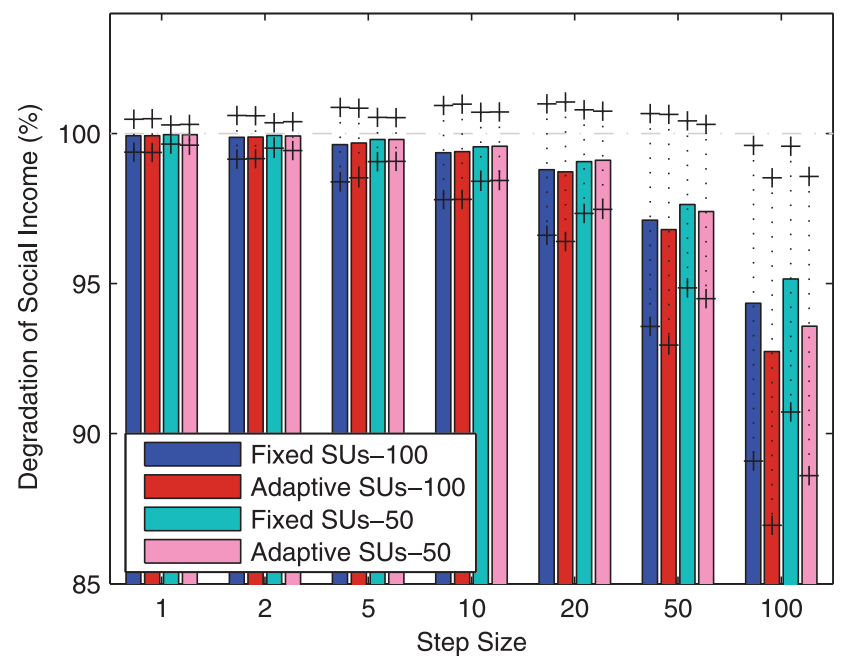

Fig. 18. Ratio of $S$ to $S^{*}$ versus step $\epsilon$, using $M=4, m_{i}=6$, $N=\{50,100\}$.

with the increasing of the number of SUs. For example, for PO4, the probability of $p_{4} \geq 2,250$ is 40,70 , and 90 percent respectively for $N=\{150,200,250\}$. This is due to the fact that a higher demand usually induces a more intensive competition among the buyers and accordingly results in a higher market price, which coincides with the common sense. Further, we can see that the equilibrium prices of POs are different, i.e., $p_{1}<\cdots<p_{4}$, due to the difference in bandwidth. This is also rational because a better item may attract more SUs and accordingly induces a more intensive competition. Fig. 20 presents the expectation of the equilibrium prices in different number of SUs using fixed step $\epsilon=10$, from which we can find a similar discovery.

Fig. 21 presents the expectations of the achieved social income (total profit) and the profit shared by POs in different number of SUs, using fixed step $\epsilon=10$. Note that we adopt the trading price solution as the pricing mechanism, that is, each PO $i$ charges the related SUs his trading price (i.e., $p_{i}$ ). The Nash bargaining solution, which always divides equally the total profit among the POs and

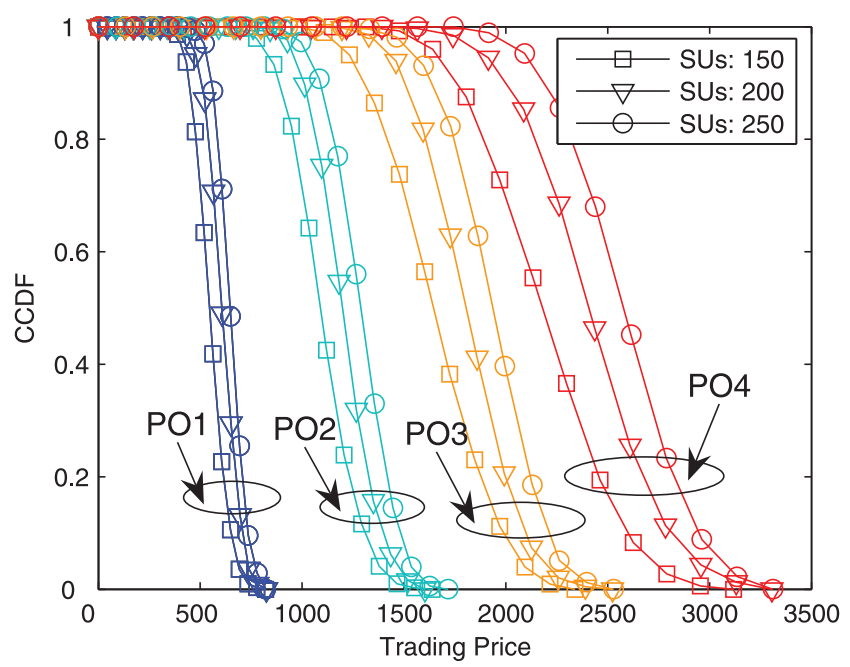

Fig. 19. CCDF of equilibrium prices, using $M=4, m_{i}=6, N=$ $\{150,200,250\}$ and fixed step $\epsilon=10$.

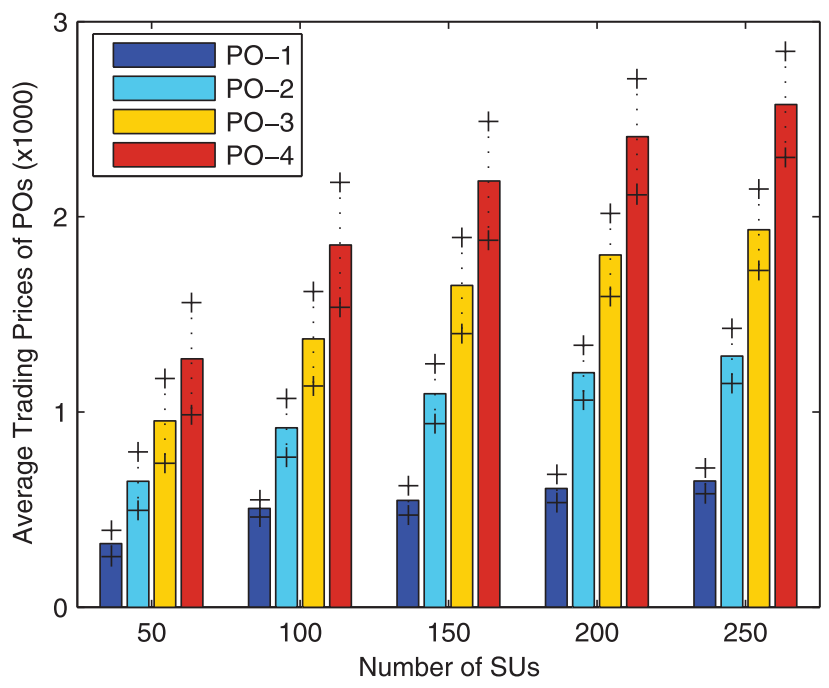

Fig. 20. Expectation of equilibrium prices versus $N$, using $M=4, m_{i}=6$ and fixed step $\epsilon=10$.

SUs, is shown as the dashed stairs in Fig. 21. As can be seen from the figure, the proportion of the profit of POs in the total profit increases from about 50 to 85 percent, as the number of SUs varies from 50 to 250 . This is due to the fact that the more SUs who competing for channels, the more profit POs can gain by raising their trading prices. In fact, as long as the number of SUs is large enough, the POs will absorb almost all of the social income. From Fig. 21 we can also suppose that, in a low demand network (e.g., $N \leq 50$ ), the profits of POs are less than those of SUs. In fact, as the total demand is the double of the total supply, i.e., $N \approx 2 \sum_{i} m_{i}$, the POs and SUs share approximately the same profits.

\subsection{Throughput}

We have studied in detail the characteristics of MAP including the converging speed, convergence probability, efficiency and profit division. Note that all above contents are related to the mechanism of MAP, while have nothing to do with the implementation of MAP in practical networks. In

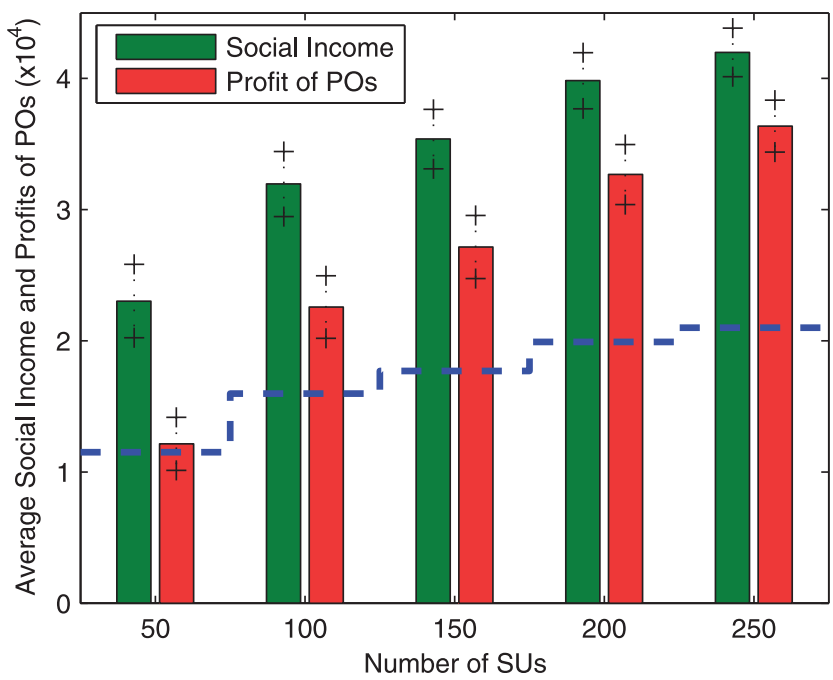

Fig. 21. Expectation of social income and profit of POs versus $N$, using $M=4, m_{i}=6$ and fixed step $\epsilon=10$. 


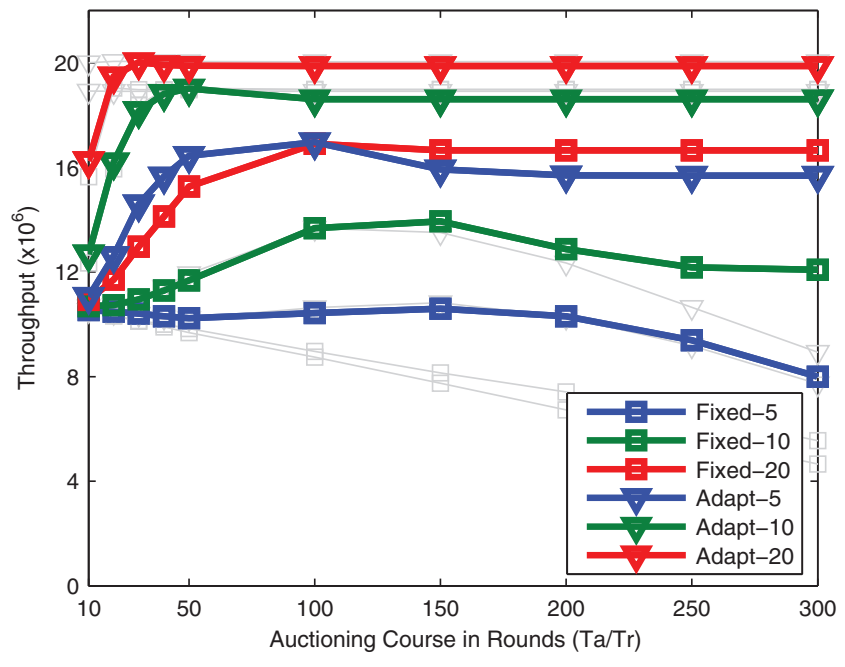

Fig. 22. Average throughput versus $T_{a}$, using $M=4, N=200, m_{i}=6$.

this section, we implement the MAP protocol in Section 5.6 in a simulation network, and we analyze the performance of MAP in different network configurations. Note that although we implement the protocol in a simulation network, it can be easily applied in a practical networks as adopting the appropriate physical and MAC layers.

As mentioned previously, if the auction ends within the period of auctioning course $T_{a}$, the remanent period of auctioning course is immediately turned into serving course. While if the auction has not achieved the equilibrium at the end of auctioning course, it will be forced to terminate. Thus we can define the throughput in each auction duration as:

$$
C=S^{\prime} \times\left(T_{s}+\left[T_{a}-T \cdot T_{r}\right]^{+}\right),
$$

where $T$ is the last round of MAP, and $S^{\prime}$ is the achieved social income. Obviously that $S^{\prime}=S$ if $T_{a} \geq T \cdot T_{r}$ and $S_{T_{a}}$ otherwise, where $S$ is the social income achieved by MAP, and $S_{T_{a}}$ is the social income at time $T_{a}$ where the auction has not yet converged to equilibrium and each PO $i$ randomly chooses $m_{i}$ SUs for serving.

Fig. 22 presents the average throughput of 1,000 auctions versus different length of auctioning course $\left(T_{a}\right)$, from which we can see that the throughput in adaptive step schemes is always greater than that in fixed step schemes. This is due to the fact that adaptive step schemes reduce the converging rounds dramatically with a tiny cost of social income degradation. Further we can see that as $T_{a}<100 \cdot T_{r}$, the throughput is strongly influenced by $T_{a}$, while as $T_{a}>100 \cdot T_{r}$, the throughput remains almost unchanged. This can be explained as follows: When $T_{a}$ is small, the achieved social income $S^{\prime}$ is like to be $S_{T_{a}}$ which is closely related to the length of auctioning course $T_{a}$. While as $T_{a}$ becomes greater, $S^{\prime}$ is like to be $S$ since the auction is likely to complete within $T_{a}$. In particular, if $T_{a}>T \cdot T_{r}$ which means the auction completes within $T_{a}$, the throughput will keep constant, i.e., $S \times\left(T_{e}-T \cdot T_{r}\right)$, that is, it is independent of the length of auctioning course. From Fig. 22 we can also find that the auction with large step is likely to achieve this situation (e.g., $T_{a} / T_{r}=150$ and 100, respectively, for the fixed steps $\epsilon=10$ and $\epsilon=20$ ).

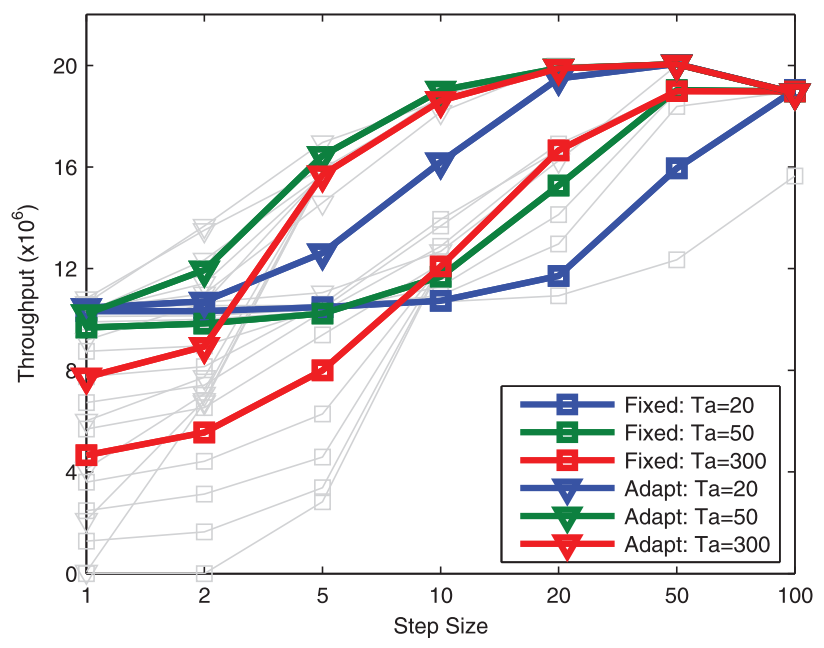

Fig. 23. Average throughput versus step $\epsilon$, using $M=4, N=200$, $m_{i}=6$.

Fig. 23 presents the average throughput versus different step schemes and step sizes, from which we can also see that the throughput in adaptive step schemes is greater than that in fixed step schemes. Further, for small $T_{a}$, the throughput increases mildly with the increasing of step size, while for large $T_{a}$, the throughput increases more acutely. This can be explained as follows: For small $T_{a}$, the auction is difficult to complete within $T_{a}$ even if the step size is large, thus the throughput increases mildly. While for large $T_{a}$, the auction is easy to complete within $T_{a}$ and a large step can reduce the converging rounds distinctly, and thus increase the serving time and the throughput. We can also see that the throughput in different $T_{a}$ converges to a particular point as the step size becomes very large. This is due to the fact that with the increasing of the step size $\epsilon$, the reduction of converging rounds becomes small since they are in approximately inverse proportion.

\section{Conclusion}

In this paper, we study the problem of residual channel allocation among SUs in CR networks with multiple POs. We propose an auction-based mechanism MAP, in which each PO systematically raises the trading prices and each SU subsequently decides whether to buy a spectrum band and from which PO he is going to buy a spectrum band. We show analytically that MAP converges to the equilibrium, and we prove that MAP achieves the optimal spectrum assignment using dual theory. We further show that, through the inherent profit transfer process in auction mechanism, both POs and SUs are willing to accept the assignment achieved by MAP. Furthermore, We discuss in detail the implementation of MAP protocol in practical networks, and we propose extensive simulation results which well coincide with the analysis.

Our proposed MAP can be applied in the CR networks without spectrum reuse among SUs, e.g., the networks within any small region or the network in wide region when the SUs work as virtual primary users and access the network through the primary base stations. Yet in practice the networks with spectrum reuse is more general. The spectrum auction with spectrum reuse is still 
a challenge for the networks with multiple sellers. We are focusing on the research of auction-based spectrum allocation with spectrum reuse in our new project.

\section{ACKNOWLEDGMENTS}

This work was supported by National Fundamental research grants (2011CB302701, 2010CB731803, and 2009CB3020402), NSF China (No. 60702046, 60832005, 60972050, and 60632040); the China Ministry of Education (No. 20070248095); the China Ministry of Education Fok Ying Tung Fund (No. 122002); a Qualcomm Research Grant; the China International Science and Technology Cooperation Programm (No. 2008DFA11630); PUJIANG Talents (08PJ14067); Shanghai Innovation Key Project (08511500400); the National Key Project of China (2009ZX03003-006-03, 2009ZX03002-003, and 2009ZX03002-005); and the National High tech grant of China (2009AA01Z248, 2009AA1Z249, and 2009AA011802).

\section{REFERENCES}

[1] T.S. Rappaport, Wireless Communication. Prentice Hall, Inc., 1996.

[2] J. Mitola, Software Radios: Wireless Architecture for the 21st Century. Wiley, 2000.

[3] J. Mitola, Cognitive Radio. John Wiley and Sons, 2006.

[4] I.F. Akyildiz, W.-Y. Lee, M.C. Vuran, and S. Mohanty, "Net Generation/Dynamic Spectrum Access/Cognitive Radio Wireless Networks: A Survey," J. Computer and Telecomm. Networking, vol. 50, pp. 2127-2159, Sept. 2006.

[5] Q. Zhao and B.M. Sadler, "A Survey of Dynamic Spectrum Access: Signal Processing, Networking, and Regulatory Policy," IEEE Signal Processing Magazine, vol. 24, no. 3, pp. 79-89, May 2007.

[6] C. Peng, H. Zheng, and B.Y. Zhao, "Utilization and Fairness in Spectrum Assignment for Opportunistic Spectrum Access," J. Mobile Networks and Applications, vol. 11, no. 4, Aug. 2006.

[7] L. Cao and H. Zheng, "Distributed Spectrum Allocation via Local Bargaining," Proc. IEEE Conf. Sensor and Ad Hoc Comm. and Networks (SECON '05), Sept. 2005.

[8] N. Nie and C. Comaniciou, "Adaptive Channel Allocation Spectrum Etiquitte for Cognitive Radio Networks," Mobile Networks and Applications, vol. 11, pp. 779-797, 2006.

[9] D. Niyato and E. Hossain, "Competitive Pricing for Spectrum Sharing in Cognitive Radio Networks: Dynamic Game, Inefficiency of Nash Equilibrium, and Collusion," IEEE J. Selected Areas in Comm., vol. 26, no. 1, pp. 192 - 202, Jan. 2008.

[10] Y. Xing, C.N. Mathur, M.A. Haleem, R. Chandramouli, and K.P. Subbalakshmi, "Dynamic Spectrum Access with QoS and Interference Temperature Constraints," IEEE Trans. Mobile Computing, vol. 6, no. 4, pp. 423-433, Apr. 2007.

[11] V. Krishna, Auction Theory. Academic Press, 2002.

[12] P. Cramton, Spectrum Auctions. Handbook of Telecomm. Economics, pp. 605-639. Emerald Group, 2002.

[13] J. Huang, R. Berry, and M.L. Honig, "Auction-Based Spectrum Sharing," ACM Mobile Networks and Applications J., vol. 11, no. 3, pp. 405-418, June 2006.

[14] S. Gandhi, C. Buragohain, L. Cao, H. Zheng, and S. Suri, "A General Framework for Wireless Spectrum Auctions," Proc. IEEE Symp. Dynamic Spectrum Access Networks (DySPAN '07), 2007.

[15] X.-Y. Li, P. Xu, S. Tang, and X. Chu, "Spectrum Bidding in Wireless Networks and Related," Proc. Conf. Computing and Combinatorics (COCOON '08), 2008.

[16] X. Zhou, S. Gandhi, S. Suri, and H. Zheng, "eBay in the Sky: Strategy-Proof Wireless Spectrum Auctions," Proc. ACM MobiCom, Sept. 2008.

[17] X. Zhou and H. Zheng, "TRUST: A General Framework for Truthful Double Spectrum Auctions," Proc. IEEE INFOCOM, 2009.

[18] J. Lian, Y. Liu, K. Naik, and L. Chen, "Virtual Surrounding Face Geocasting with Guaranteed Message Delivery for Ad Hoc and Sensor Networks," IEEE/ACM Trans. Networking, vol. 17, no. 1, pp. 200-211, Feb. 2009.

[19] C.H. Papadimitriou and K. Steiglitz, Combinatorial Optimization: Algorithms and Complexity. Prentice-Hall, 1982.
[20] A. Gibbons, Algorithmic Graph Theory. Cambridge Univ., 1985

[21] A. Schrijver, Theory of Linear and Integer Programming. John Wiley and Sons, 1998.

[22] J. Munkres, "Algorithms for the Assignment and Transportation Problems," J. Soc. Industrial and Applied Math., vol. 5, no. 1, pp. 3238, Mar. 1957.

[23] J. Nash, "The Bargaining Problem," Econometrica, vol. 18, no. 2, pp. 155-162, 1950.

[24] H. Yu, L. Gao, Y. Li, X. Gan, X. Wang, W. Chen, A.V. Vasilakos, and $\mathrm{Y}$. Xu, "Information Sharing in Spectrum Auction for Dynamic Spectrum Access," Proc. Conf. IEEE Global Telecomm. (GlobeCom'09), Nov. 2009.

[25] H. Yu, L. Gao, Z. Li, X. Wang, and E. Hossain, "Pricing for Uplink Power Control in Cognitive Radio Networks," IEEE Trans. Vehicular Technology, vol. 59, no. 4, pp. 1769-1778, May 2010.

[26] X. Wang, Z. Li, P. Xu, Y. Xu, X. Gao, and H. Chen, "Spectrum Sharing in Cognitive Radio Networks-An Auction-Based Approach," IEEE Trans. System, Man and Cybernetics-Part B: Cybernetics, vol. 40, no. 3, pp. 587-596, June 2010.

[27] H. Su and X. Zhang, "Cross-Layer Based Opportunistic MAC Protocols for QoS Provisionings over Cognitive Radio Mobile Wireless Networks," IEEE J. Selected Areas Comm., vol. 26, no. 1, pp. 118-129, Jan. 2008.

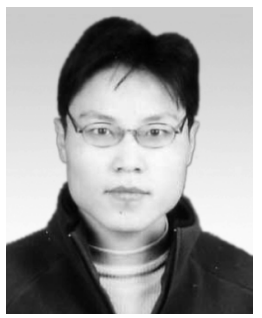

Lin Gao received the BS degree in information engineering from the Nanjing University of Posts and Telecommunications, China, in 2002, and the MS degree in electronic engineering from Shanghai Jiao Tong University, China, in 2006 Currently, he is working toward the $\mathrm{PhD}$ degree in the Institute of Wireless Communication Technology (IWCT) at Shanghai Jiao Tong University. His research interests include channel-adaptive technologies, cross-layer designs, distributed resource allocations for mobile and wireless networks, dynamic spectrum allocation and management in cognitive radio networks, and game theory and learning. $\mathrm{He}$ is a student member of the IEEE.

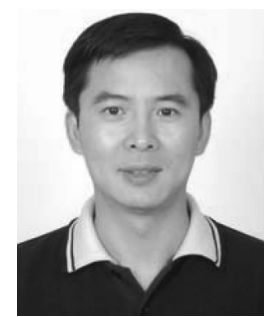

Youyun $\mathrm{Xu}$ received the $\mathrm{PhD}$ degree in information and communication engineering form Shanghai Jiao Tong University in 1999 . Currently, he is working as a professor with the Nanjing Institute of Communication Engineering, China. He is also a part time professor in the Institute of Wireless Communication Technology at Shanghai Jiao Tong University, China. He has more than 20 years professional experience in teaching and researching in communication theory and engineering. Now, his research interests are focussed on new generation wireless mobile communication systems (IMT-advanced and related), advanced channel coding and modulation techniques, multiuser information theory and radio resource management, wireless sensor networks, and cognitive radio networks. He is a member of the IEEE and a senior member of the Chinese Institute of Electronics.

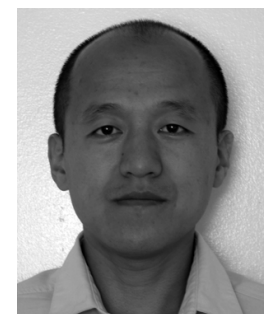

Xinbing Wang received the BS degree (with hons.) from the Department of Automation, Shanghai Jiaotong University, China, in 1998, and the MS degree from the Department of Computer Science and Technology, Tsinghua University, Beijing, China, in 2001. He received the $\mathrm{PhD}$ degree in electrical and computer engineering with a minor in mathematics from North Carolina State University, Raleigh, in 2006. Currently, he is a faculty member in the Department of Electronic Engineering, Shanghai Jiaotong University, China. His research interests include resource allocation and management in mobile and wireless networks, TCP asymptotics analysis, wireless capacity, cross layer call admission control, asymptotics analysis of hybrid systems, and congestion control over wireless ad hoc and sensor networks. He has been a member of the technical program committees of several conferences including IEEE INFOCOM 2009-2011, IEEE ICC 2007-2011, and IEEE Globecom 2007-2011. He is a member of the IEEE. 